\title{
Structural evolution in germanium and selenium nuclei within the mapped interacting boson model based on the Gogny energy density functional
}

\author{
K. Nomura, ${ }^{1,2}$ R. Rodríguez-Guzmán, ${ }^{3}$ and L. M. Robledo ${ }^{4}$ \\ ${ }^{1}$ Physics Department, Faculty of Science, University of Zagreb, HR-10000 Zagreb, Croatia \\ ${ }^{2}$ Center for Computational Sciences, University of Tsukuba, Tsukuba 305-8577, Japan \\ ${ }^{3}$ Physics Department, Kuwait University, 13060 Kuwait, Kuwait \\ ${ }^{4}$ Departamento de Física Teórica, Universidad Autónoma de Madrid, E-28049 Madrid, Spain \\ (Received 16 February 2017; revised manuscript received 3 April 2017; published 13 June 2017)
}

\begin{abstract}
The shape transitions and shape coexistence in the Ge and Se isotopes are studied within the interacting boson model (IBM) with the microscopic input from a self-consistent mean-field calculation based on the Gogny-D1M energy density functional. The mean-field energy surface as a function of the quadrupole shape variables $\beta$ and $\gamma$, obtained from the constrained Hartree-Fock-Bogoliubov method, is mapped onto the expectation value of the IBM Hamiltonian with configuration mixing in the boson condensate state. The resultant Hamiltonian is used to compute excitation energies and electromagnetic properties of the selected nuclei ${ }^{66-94} \mathrm{Ge}$ and ${ }^{68-96} \mathrm{Se}$. Our calculation suggests that many nuclei exhibit $\gamma$ softness. Coexistence between prolate and oblate shapes, as well as between spherical and $\gamma$-soft shapes, is also observed. The method provides a reasonable description of the observed systematics of the excitation energy of the low-lying energy levels and transition strengths for nuclei below the neutron shell closure $N=50$, and provides predictions on the spectroscopy of neutron-rich Ge and $\mathrm{Se}$ isotopes with $52 \leqslant N \leqslant 62$, where data are scarce or not available.
\end{abstract}

DOI: 10.1103/PhysRevC.95.064310

\section{INTRODUCTION}

The evolution of nuclear shapes has attracted considerable interest in nuclear structure studies [1-3]. In particular, the precise description of the structural evolution along different isotopic and/or isotonic chains as well as the associated shell effects require an accurate modeling of the nuclear many-body problem. Within this context, the germanium and selenium nuclei belong to one of the most challenging regions of the nuclear chart. Their structure and decay patterns have been extensively studied in recent years both experimentally [4-7] and theoretically [8-16]. Among the theoretical approximations used to study those nuclei are the shell model (SM) [8-10], the energy density functional (EDF) framework [11-14], and the algebraic approach $[15,16]$. The shape transitions in the neighborhood of the neutron subshell closure $N=40$ have also received considerable attention $[6,7,12,13]$. Moreover, the Ge and Se nuclei have been shown to exhibit a pronounced competition between different configurations associated with a variety of intrinsic shapes, i.e., shape coexistence [17]. The corresponding spectra display low-lying excited $0^{+}$energy levels which could be linked to proton intruder excitations across the $Z=28$ shell gap.

The EDF framework is among the most popular tools employed in microscopic nuclear structure studies. It allows a description of the properties of the bulk nuclear matter and the ground states of finite nuclei all over the nuclear chart [18]. Calculations are usually carried out in terms of the nonrelativistic Skyrme [18,19] and Gogny [20] EDFs but also within the relativistic mean-field (RMF) approximation $[21,22]$. On the one hand, the mean-field approximation has already been successfully applied to nuclei with mass number $A \approx 70-100$ [7,11-14,23,24]. On the other hand, the quantitative analysis of the collective excitations in those systems requires the inclusion of correlations not explicitly taken into account within the mean-field picture. Those correlations stem from the restoration of the symmetries (spontaneously) broken at the mean-field level and/or the fluctuations in the collective coordinates. They are usually taken into account within the symmetry-projected generator coordinate method (GCM) $[18,22,25,26]$. The symmetry projected GCM offers a reasonable starting point to account for the dynamical interplay between the single-particle and collective degrees of freedom in atomic nuclei. However, the calculations are highly demanding from a computational point of view, especially in those cases where several collective coordinates should be included in the GCM ansatz. Therefore, an expansion in the nonlocality of the norm and Hamiltonian kernels is used to build a collective Hamiltonian approach [27] that alleviates the computational burden. At this point the few-determinant (FED) excited VAMPIR approach of the Tubingen group, used to describe shape coexistence in some Ge and Se isotopes in [28-32], has to be mentioned.

In this study, we have resorted to the fermion-to-boson mapping procedure introduced in Ref. [33] as an alternative approach to describe the considered Ge and Se nuclei. The method maps the (fermionic) energy surfaces obtained with constrained mean-field calculations onto the bosonic ones computed as the expectation value of the interacting boson model (IBM) [34] Hamiltonian in the boson coherent state. By the mapping procedure, the parameters of the IBM Hamiltonian for each individual nucleus are completely determined, i.e., no phenomenological adjustment of the parameters to the experimental data is required. The IBM Hamiltonian is then diagonalized and the resulting wave functions are used to compute the spectroscopic properties of ${ }^{66-94} \mathrm{Ge}$ and ${ }^{68-96} \mathrm{Se}$. The fermion-to-boson mapping procedure has allowed an accurate, computationally economic and systematic description of the 
shape coexistence [35], the structural evolution in $A \approx 100$ nuclei [36], and the quadrupole and octupole transitions in the light actinide and rare-earth regions [37,38] as well as odd-mass nuclei [39]. In this work, we demonstrate the ability of the mapping scheme to account for the properties of the nuclei on the neutron-deficient side $(N \leqslant 50)$, where there are enough experimental data to compare with. So far, the IBM has been used in phenomenological studies of Ge and Se nuclei $[15,40,41]$. However, one of the advantages of our approach is that it is able to provide predictions for unexplored regions. We then extrapolate the method to neutron-rich nuclei with $N=52-62$, for which experimental data are not available. The microscopic input is provided by constrained HartreeFock-Bogoliubov (HFB) calculations based on the finite range and density-dependent Gogny EDF [20]. In particular, we have employed the parametrization D1M [42]. Previous studies have shown that the parametrization D1M essentially keeps the same predictive power as the well tested Gogny-D1S [43] EDF to describe a wealth of low-energy nuclear structure phenomena.

The paper is organized as follows. The theoretical framework used in our calculations is outlined in Sec. II. The meanfield and mapped energy surfaces are discussed in Sec. III while the derived IBM parameters are presented in Sec. IV. We then discuss in Sec. V the evolution of the low-lying levels in the considered nuclei, as well as the systematics of the $B(E 2)$ transition rates, spectroscopic quadrupole moments, and monopole transition rates. We also discuss the individual level schemes for the $N=38,40,42$, and 60 isotones, which are representative cases of the $\gamma$ softness and/or shape coexistence. In Sec. VI, we address the sensitivity of our predictions with respect to the particular version of the Gogny EDF employed in the calculations. Finally, Sec. VII is devoted to the conclusions and work prospects.

\section{DESCRIPTION OF THE MODEL}

\section{A. Self-consistent mean-field calculations}

As a first step, we have performed (constrained) HFB calculations based on the Gogny EDF. They provide the deformation energy surfaces for the considered $\mathrm{Ge}$ and $\mathrm{Se}$ nuclei as functions of the corresponding quadrupole deformation parameters. We have used constrains on the multipole operators $\hat{Q}_{20}$ and $\hat{Q}_{22}[44,45]$, which are associated with the deformation parameters $\beta$ and $\gamma$ [1] in such a way that $\beta=\sqrt{4 \pi / 5} Q /\left\langle r^{2}\right\rangle$ and $\gamma=\tan ^{-1} Q_{22} / Q_{20}$. Note that $Q=\sqrt{Q_{20}^{2}+Q_{22}^{2}}$ is the intrinsic quadrupole moment while $\left\langle r^{2}\right\rangle$ represents the mean-square radius obtained from the HFB state. For a more detailed account, the reader is referred to Ref. [45]. In what follows we will refer to the set of HFB energies, as functions of the deformation parameters $\beta$ and $\gamma$, as the (mean-field) energy surface.

\section{B. The IBM Hamiltonian}

To describe the spectroscopic properties of the studied nuclei, we have resorted to the fermion-to-boson mapping procedure introduced in Ref. [33]. Within such a scheme, the (fermionic) energy surface obtained at the Gogny-HFB level for a given nucleus is mapped onto the expectation value of the IBM Hamiltonian in the boson coherent state [46]. The parameters of the IBM Hamiltonian are then determined by this procedure and the excitation energies as well as the IBM wave functions are determined via the diagonalization of the mapped Hamiltonian. The transition rates are computed using such IBM wave functions.

Our IBM model comprises the collective nucleon pairs in the valence space with spin and parity $J^{\pi}=0^{+}$(monopole $S$ pair) and $2^{+}$(quadrupole $D$ pair). They are associated with the $J^{\pi}=0^{+}(s)$ and $2^{+}(d)$ bosons, respectively [47]. The total number of bosons, denoted by $N_{B}$, amounts to half the number of valence nucleons. In this study, the IBM configuration space comprises the proton $Z=28-50$ major shell as well as the two neutron major shells $N=28-50$ and $N=50-82$. Therefore, $2 \leqslant N_{B} \leqslant 7\left(3 \leqslant N_{B} \leqslant 8\right)$ and $3 \leqslant N_{B} \leqslant 8\left(4 \leqslant N_{B} \leqslant 9\right)$ for ${ }^{66-82} \mathrm{Ge}\left({ }^{84-94} \mathrm{Ge}\right)$ and ${ }^{68-84} \mathrm{Se}\left({ }^{86-96} \mathrm{Se}\right)$, respectively. In this study, for the sake of simplicity, no distinction has been made between the proton and neutron degrees of freedom.

As will be shown later, the Gogny-HFB energy surfaces, for many of the considered nuclei, exhibit two minima close in energy. Within the mean-field picture, such minima can be associated with the normal $0 \mathrm{p}-0 \mathrm{~h}$ and intruder $2 \mathrm{p}-2 \mathrm{~h}$ excitations across the shell gap. In the present case, we assume that the intruder configuration corresponds to the proton $2 \mathrm{p}-2 \mathrm{~h}$ excitation across the shell closure $Z=28$. To account for the intruder configuration, the boson model space has to be extended. Duval and Barrett [48] proposed a method that incorporates the intruder configurations by introducing several independent IBM Hamiltonians. As particles and holes are usually not distinguished, the $2 \mathrm{p}-2 \mathrm{~h}$ excitation increases the boson number by 2 . The different shell-model-like spaces of $2 n \mathrm{p}-2 n \mathrm{~h}(n=0,1)$ configurations can be then associated with the corresponding boson spaces comprising $N_{B}+2 n$ bosons. The different boson configuration spaces are allowed to mix via certain mixing interaction.

The Hilbert space of the configuration mixing IBM model is then defined as the direct sum of each unperturbed Hamiltonian, i.e., $\left[N_{B}\right] \oplus\left[N_{B}+2\right]$, where $\left[N_{B}+2 n\right]$ denotes the unperturbed space corresponding to the $2 n \mathrm{p}-2 n \mathrm{~h}$ configurations comprising $N_{B}+2 n$ bosons. In what follows, we will simply denote the configuration $\left[N_{B}+2 n\right](n=0,1)$ as $[n]$. Our criterion to include the configuration mixing for a given nucleus is that the second-lowest minimum in the mean-field energy surface is clear enough so as to constrain the corresponding unperturbed Hamiltonian for the intruder configuration. According to this criterion the configuration-mixing has been taken into account for the nuclei ${ }^{66,70-74,90-94} \mathrm{Ge}$ and ${ }^{68-76,90-96} \mathrm{Se}$ in this paper.

We have resorted to the configuration-mixing IBM Hamiltonian [48]

$$
\hat{H}=\hat{H}_{0}+\left(\hat{H}_{1}+\Delta\right)+\hat{H}_{\mathrm{mix}},
$$

where $\hat{H}_{n}(n=0,1)$ is the Hamiltonian for the unperturbed configuration $[n]$ while $\hat{H}_{\text {mix }}$ stands for the interaction mixing both spaces. In Eq. (1), $\Delta$ represents the energy needed to excite one boson from one major shell to the next. 

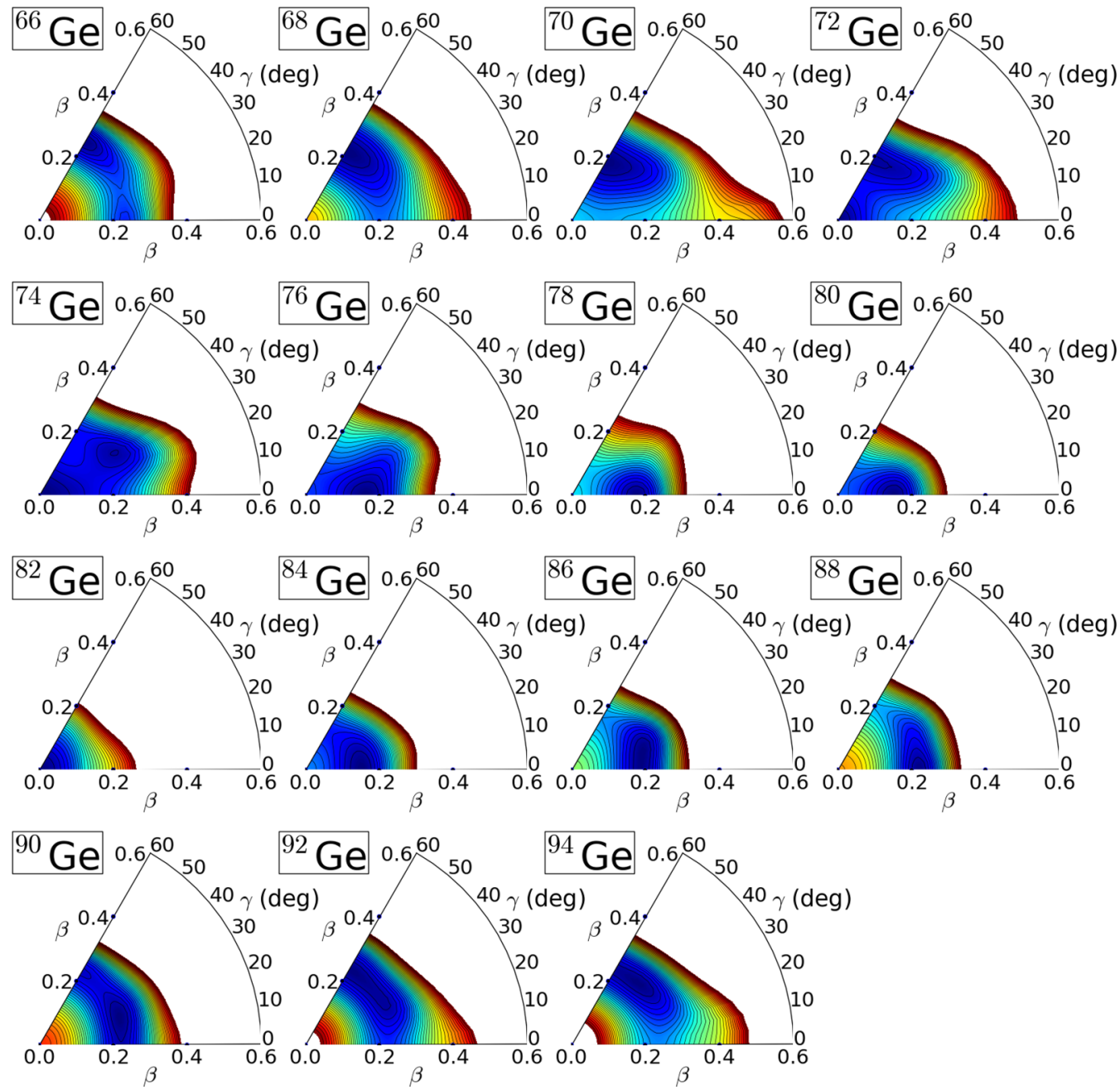

FIG. 1. Mean-field energy surfaces for the nuclei ${ }^{66-94}$ Ge. Results have been obtained with the Gogny-D1M EDF. The energy difference between neighboring contours is $100 \mathrm{keV}$.

For each configuration space, we have employed the simplest form of the IBM-1 Hamiltonian that still simulates the essential ingredients of the low-energy quadrupole dynamics, i.e.,

$$
\hat{H}_{n}=\epsilon_{n} \hat{n}_{d}+\kappa_{n} \hat{Q} \cdot \hat{Q}+\kappa_{n}^{\prime} \hat{V}_{d d d} .
$$

The first term $\hat{n}_{d}=d^{\dagger} \cdot \tilde{d}$ in Eq. (2) is the $d$-boson number operator, and $\epsilon_{n}$ is the single $d$-boson energy in the $[n]$ space. The second term represents the quadrupole-quadrupole interaction with strength parameter $\kappa_{n}$. The quadrupole operator $\hat{Q}$ in boson space reads $\hat{Q}=s^{\dagger} \tilde{d}+d^{\dagger} s+\chi_{n}\left[d^{\dagger} \times \tilde{d}\right]^{(2)}$, where $\chi_{n}$ is a parameter. The third term stands for a specific three-body interaction among $d$ bosons, with strength $\kappa_{n}^{\prime}$, which is required to describe $\gamma$-soft systems [49]. It takes the form

$$
\hat{V}_{d d d}=\left[\left[d^{\dagger} \times d^{\dagger} \times d^{\dagger}\right]^{(L)} \times[\tilde{d} \times \tilde{d} \times \tilde{d}]^{(L)}\right]^{(0)},
$$

where the symbol $\times$ represents a tensor coupling and $L$ is the total angular momentum of the boson system. In our calculations, we have only included the term with $L=3$ as it gives rise to a stable minimum at $\gamma \approx 30^{\circ}$. The mixing interaction term $\hat{H}_{\text {mix }}$ reads

$$
\hat{H}_{\text {mix }}=\omega_{s} s^{\dagger} s^{\dagger}+\omega_{d} d^{\dagger} \cdot d^{\dagger}+(\text { H.c. }),
$$

where $\omega_{s}$ and $\omega_{d}$ are strength parameters. For simplicity, we have assumed $\omega_{s}=\omega_{d} \equiv \omega$.

To associate a Gogny-HFB energy surface with the corresponding configuration-mixing IBM Hamiltonian Eq. (1), an extended boson coherent state

$$
|\Phi(\beta, \gamma)\rangle=\left|\Phi\left(N_{0}, \beta, \gamma\right)\right\rangle \oplus\left|\Phi\left(N_{1}, \beta, \gamma\right)\right\rangle,
$$

has been introduced with $N_{n}=N_{B}+2 n(n=0,1)$. For each unperturbed configuration space $\left|\Phi\left(N_{n}, \beta, \gamma\right)\right\rangle(n=0,1)$, the coherent state is taken in the form

$$
\left|\Phi\left(N_{n}, \beta, \gamma\right)\right\rangle=\frac{1}{\sqrt{N_{n} !}}\left(\lambda^{\dagger}\right)^{N_{n}}|0\rangle,
$$

where $|0\rangle$ denotes the inert core and

$$
\lambda^{\dagger}=s^{\dagger}+\beta_{B} \cos \gamma_{B} d_{0}^{\dagger}+\frac{1}{\sqrt{2}} \beta_{B} \sin \gamma_{B}\left(d_{+2}^{\dagger}+d_{-2}^{\dagger}\right) .
$$



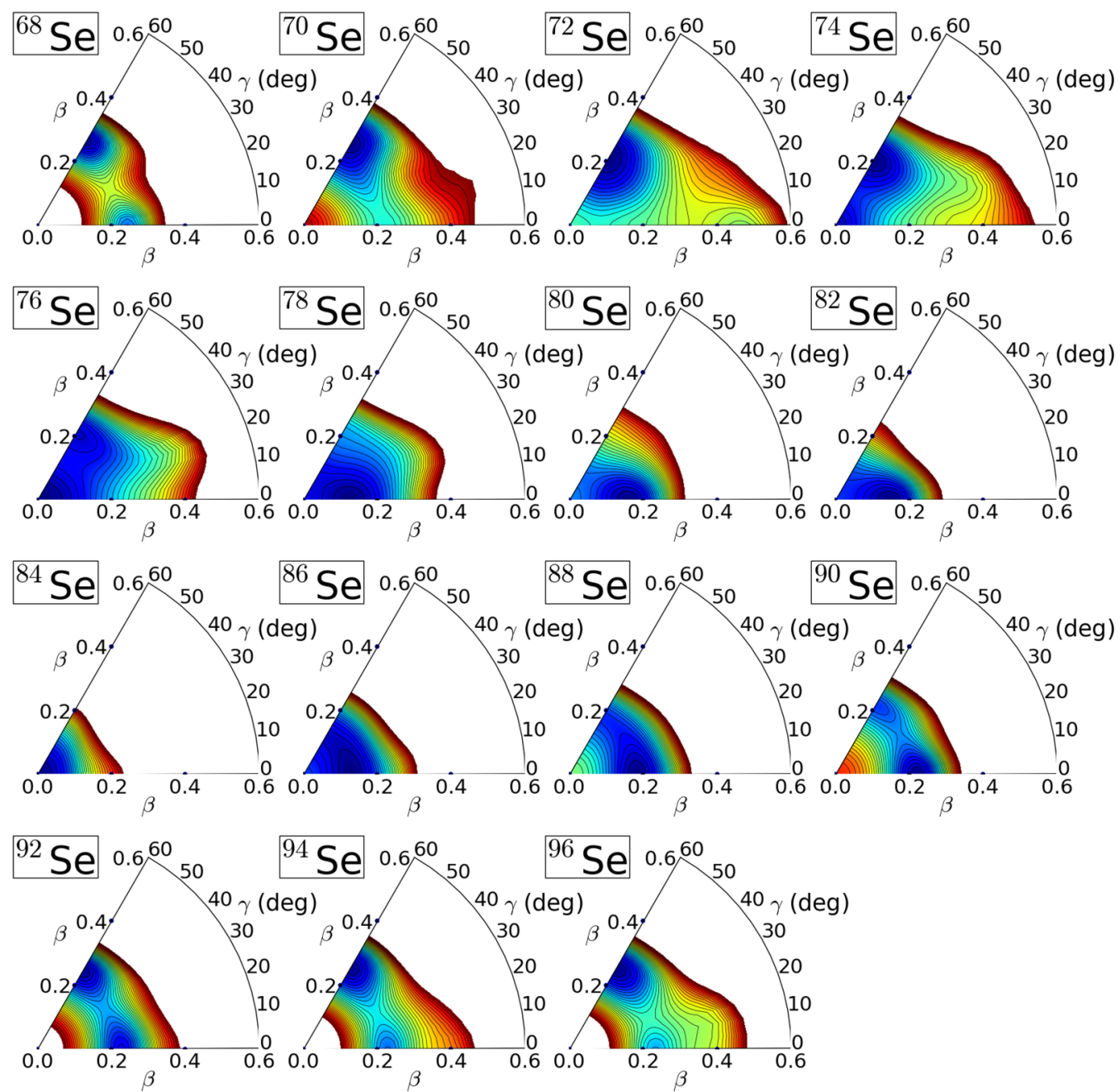

FIG. 2. The same as in Fig. 1, but for the nuclei ${ }^{68-96} \mathrm{Se}$.

On the other hand, $\beta_{B}$ and $\gamma_{B}$ are the boson analogs of the quadrupole deformation parameters $\beta$ and $\gamma$ within the geometrical collective model [1].

The expectation value of the total Hamiltonian $\hat{H}$ in the coherent state $|\Phi(\beta, \gamma)\rangle$ leads to a $2 \times 2$ matrix [50]:

$$
\mathcal{E}=\left(\begin{array}{cc}
E_{0}(\beta, \gamma) & \Omega(\beta) \\
\Omega(\beta) & E_{1}(\beta, \gamma)+\Delta
\end{array}\right),
$$

with diagonal and off-diagonal elements accounting for the expectation values of the unperturbed and mixing terms, respectively. The two eigenvalues of $\mathcal{E}$ correspond to specific energy surfaces. It is customary to take the lower-energy one [50] as the $\operatorname{IBM}(\beta, \gamma)$ energy.

The diagonal matrix element $E_{n}(\beta, \gamma)$ is given by

$$
\begin{aligned}
E_{n}(\beta, \gamma)= & \frac{k_{1}+k_{2} \beta_{n}^{2}}{1+\beta_{n}^{2}}+\frac{k_{3} \beta_{n}^{2}+k_{4} \beta_{n}^{3} \cos 3 \gamma+k_{5} \beta_{n}^{4}}{\left(1+\beta_{n}^{2}\right)^{2}} \\
& +\frac{k_{6} \beta_{n}^{6} \sin ^{2} 3 \gamma}{\left(1+\beta_{n}^{2}\right)^{3}},
\end{aligned}
$$

where $k_{1}=5 \kappa_{n} N_{n}, k_{2}=\left[\epsilon_{n}+\kappa_{n}\left(1+\chi_{n}^{2}\right)\right] N_{n}, k_{3}=4 \kappa_{n} N_{n}\left(N_{n}-1\right)$, $k_{4}=-4 \kappa_{n} \sqrt{2 / 7} N_{n}\left(N_{n}-1\right) \chi_{n}, k_{5}=(2 / 7) \kappa_{n} N_{n}\left(N_{n}-1\right) \chi_{n}^{2}$, and $\quad k_{6}=(1 / 30) \kappa_{n}^{\prime} N_{n}\left(N_{n}-1\right)\left(N_{n}-2\right)$. Moreover, the nondiagonal matrix element reads

$$
\Omega(\beta)=\omega \sqrt{\left(N_{B}+1\right)\left(N_{B}+2\right)}\left[\frac{1+\beta_{0} \beta_{1}}{\sqrt{\left(1+\beta_{0}^{2}\right)\left(1+\beta_{1}^{2}\right)}}\right]^{N_{B}} .
$$

Note that, in Eqs. (9) and (10), $\beta_{n}$ represents the bosonic deformation parameter for each unperturbed space $[n]$. It is related to the Gogny-HFB one as $\beta_{n}=C_{n} \beta$. The constant $C_{n}$ is also determined by fitting the (fermionic) Gogny-HFB energy surface to the (bosonic) IBM one. To this end, one requires that the position of the minimum, for each unperturbed configuration, be reproduced. Both Eqs. (9) and (10) are similar to the ones employed in our previous studies $[36,51,52]$ within the IBM-2 framework.

\section{Derivation of the IBM parameters: The fitting procedure}

The Hamiltonian in Eq. (1) contains ten parameters. They have been determined along the following lines: 

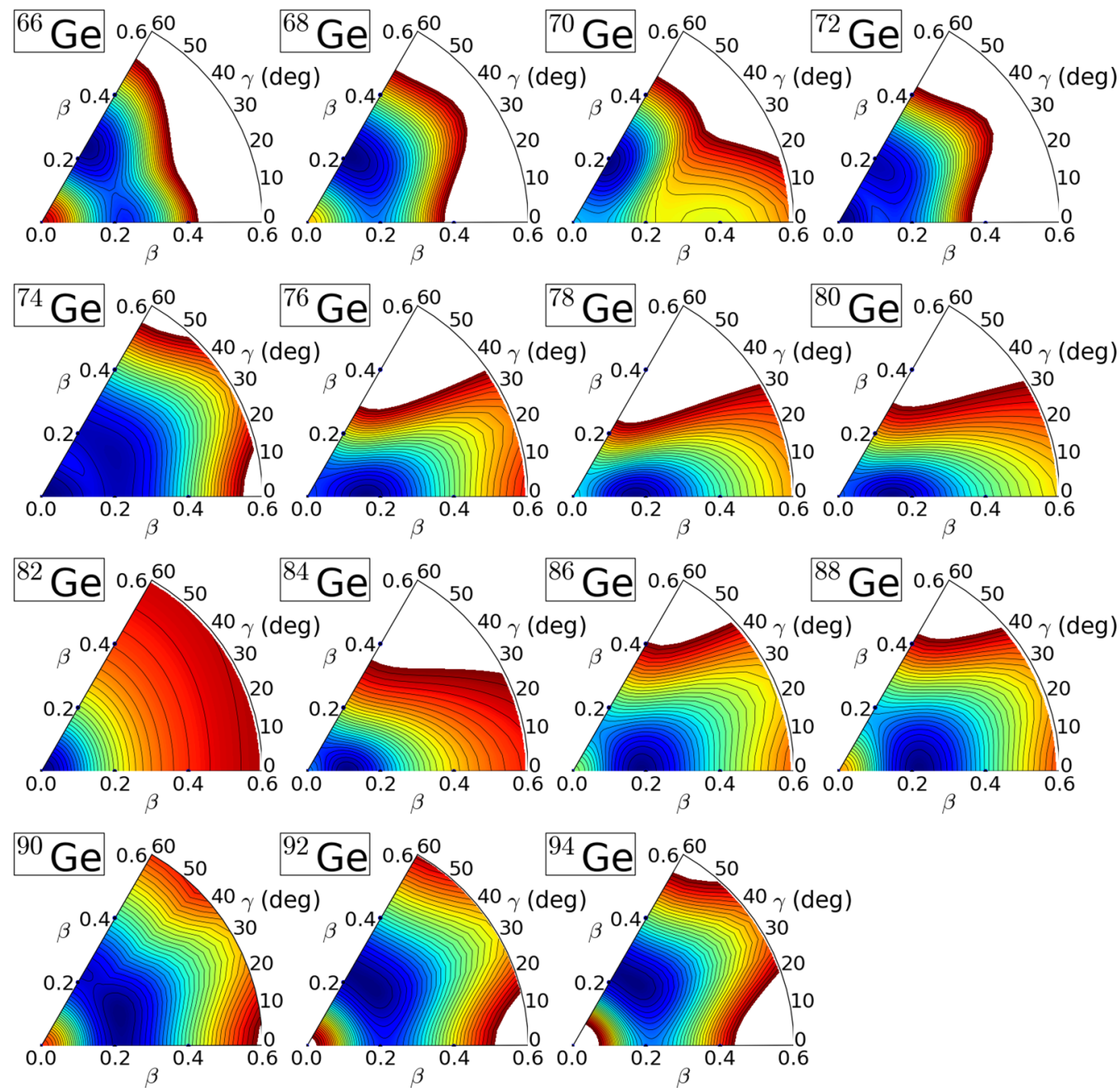

FIG. 3. The same as in Fig. 1, but for the mapped IBM energy surfaces.

Step 1: Each unperturbed Hamiltonian is determined by using the procedure of Refs. [33,36,53]. Here, each diagonal matrix element $E_{n}$ in Eq. (8) is fitted to the corresponding mean-field minimum. The normal $[n=0]$ configuration is assigned to the mean-field minimum with the smallest deformation while the $[n=1]$ configuration is assigned to the HFB minimum with the larger deformation. In this way, each unperturbed Hamiltonian is determined independently.

Step 2: The energy offset $\Delta$ is determined so that the energy difference between the two minima (Step 1) of the Gogny-HFB energy surface is reproduced.

Step 3: Finally, the strength parameter $\omega$ of the mixing interaction term $\hat{H}_{\text {mix }}$ is determined so as to reproduce the shapes of the barriers between the minima $[51,52]$.

In Step 1, note that the link of the $0 \mathrm{p}-0 \mathrm{~h}$ and $2 \mathrm{p}$ $2 \mathrm{~h}$ configurations with the small and large deformation minima, respectively, is based on the assumption that the well-established interpretation of shape coexistence in the neutron-deficient lead region [54-56] also holds here. In these references, the $0_{1}^{+}$ground state is associated with a weakly deformed oblate shape and the intruder $\mathrm{O}_{2}^{+}$state with a prolate shape with larger deformation.

Once the IBM parameters for each of the considered nuclei are determined, the Hamiltonian $\hat{H}$ is diagonalized in the $[0] \oplus[1]$ space by using the code IBM-1 [57]. The IBM wave functions resulting from the diagonalization are then used to compute electromagnetic properties that could be considered as signatures of shape coexistence and/or shape transitions, such as the $B(E 2)$ transition probabilities, the spectroscopic quadrupole moments $Q_{s p}$, and the $\rho^{2}(E 0)$ values between $0^{+}$ states. The $B(E 2)$ transition probabilities read

$$
B\left(E 2 ; J_{i} \rightarrow J_{f}\right)=\frac{1}{2 J_{i}+1}\left|\left\langle J_{f}\left\|\hat{T}^{(E 2)}\right\| J_{i}\right\rangle\right|^{2},
$$

where $J_{i}$ and $J_{f}$ are the spins of initial and final states, respectively. On the other hand, the spectroscopic quadrupole moments and the $\rho^{2}(E 0)$ values are computed as

$$
Q_{s p}=\sqrt{\frac{16 \pi}{5}}\left(\begin{array}{ccc}
J & 2 & J \\
-J & 0 & J
\end{array}\right)\left\langle J\left\|\hat{T}^{(E 2)}\right\| J\right\rangle
$$


and

$$
\rho^{2}\left(E 0 ; 0_{i}^{+} \rightarrow 0_{f}^{+}\right)=\frac{Z^{2}}{R_{0}^{4}}\left|\left\langle 0_{f}^{+}\left\|\hat{T}^{(E 0)}\right\| 0_{i}^{+}\right\rangle\right|^{2},
$$

where $R_{0}=1.2 A^{1 / 3} \mathrm{fm}$. The $E 0$ and $E 2$ operators take the form $\hat{T}^{(E 0)}=\sum_{n=0,1} e_{0, n} \hat{n}_{d}$ and $\hat{T}^{(E 2)}=\sum_{n=0,1} e_{2, n} \hat{Q}$, respectively. For the effective charges we have assumed $e_{0,0}=$ $e_{0,1} \equiv e_{0}$ and $e_{2,0}=e_{2,1} \equiv e_{2}$. Their numerical values have been fitted so as to reproduce the experimental $B\left(E 2 ; 2_{1}^{+} \rightarrow\right.$ $\left.0_{1}^{+}\right)$[58] and $\rho^{2}\left(E 0 ; 0_{2}^{+} \rightarrow 0_{1}^{+}\right)$[59] values for the $N=42$ and $N=40$ nuclei, respectively.

\section{ENERGY SURFACES}

\section{A. Gogny-D1M energy surfaces}

The Gogny-D1M energy surfaces, obtained for the nuclei ${ }^{66-94} \mathrm{Ge}$ and ${ }^{68-96} \mathrm{Se}$, are shown in Figs. 1 and 2. Similar results have been obtained with the parametrization D1S of the Gogny-EDF and therefore they will not be discussed in detail in this section. As can be seen from Fig. 1, the nucleus ${ }^{66} \mathrm{Ge}$ exhibits coexisting prolate and oblate minima with $\beta \approx 0.2$. The prolate minimum becomes less pronounced in both ${ }^{68,70} \mathrm{Ge}$. A shape transition is observed between the ground-state shapes of ${ }^{70} \mathrm{Ge}$ and ${ }^{72} \mathrm{Ge}$. For the latter, a spherical minimum emerges and becomes the ground state at the meanfield level. Such a spherical ground state could be associated with the $N=40$ neutron subshell closure. Furthermore, a close-lying oblate minimum is also observed in the energy surface of ${ }^{72} \mathrm{Ge}$. In the case of ${ }^{74} \mathrm{Ge}$, one observes a coexistence between the spherical ground state and a triaxial minimum with $\gamma \approx 30^{\circ}$. A single prolate minimum, which is notably $\gamma$-soft, is found for ${ }^{76} \mathrm{Ge}$. For higher neutron numbers, the minimum moves gradually from prolate to spherical, reflecting the proximity of the $N=50$ neutron shell closure. A prolate minimum develops from ${ }^{82} \mathrm{Ge}$ to ${ }^{88} \mathrm{Ge}$ and becomes $\gamma$-softer as a function of the neutron number. On the other hand, a shallow oblate minimum is found for ${ }^{90} \mathrm{Ge}$. An oblate and $\gamma$-soft ground state is predicted for the isotopes ${ }^{92,94} \mathrm{Ge}$. As can be seen from Fig. 2, a similar structural evolution is predicted for the studied Se nuclei. Our Gogny-D1M HFB trends agree well with previous results obtained within the relativistic mean-field (RMF) approximation [12]. A coexistence between spherical and oblate configurations has also been found for ${ }^{70,72} \mathrm{Se}[60]$ and ${ }^{74} \mathrm{Se}$ within the five-dimensional (5D) collective Hamiltonian approach based on the Gogny-D1S EDF [11].

\section{B. Mapped IBM energy surfaces}

In Figs. 3 and 4 we have plotted the IBM energy surfaces obtained by mapping the Gogny-D1M ones already shown in Figs. 1 and 2. First we realize that, compared with the mean-field energy surfaces, the IBM ones are generally more flat in those regions of the $\beta-\gamma$ plane away from the ground state minimum. This behavior arises as a consequence of the limited number of nucleon pairs (bosons) comprising the IBM valence space but also because the Hamiltonian used for each configuration space Eq. (2) is too simple to reproduce every detail of the fermionic energy surfaces $[33,53]$. In order to determine the IBM Hamiltonian we have reproduced the location and depth of the energy minimum as well as the curvatures along both the $\beta$ and $\gamma$ directions around the minimum. Furthermore, we have also reproduced the topology of the barriers separating the different minima. With this in mind, ones observes from Figs. 3 and 4 that the trends observed as functions of the neutron number $N$ in the mapped energy surfaces mimic quite well the ones found in the Gogny-D1M case.

\section{EVOLUTION OF THE DERIVED IBM PARAMETERS}

In Fig. 5 we have depicted the parameters of the IBM Hamiltonian, obtained via the fermion-to-boson mapping procedure, as functions of the neutron number. The decrease of the single $d$-boson energy $\epsilon$ [panels (a) and (b)] when moving towards the open-shell region, reflects the emergence of collectivity. For both the normal and intruder configurations, the parameter $\epsilon$ increases when approaching the neutron subshell $N \approx 40$ and the magic number $N=50$. On the other hand, the $\epsilon$ values for neutron-rich Ge and Se nuclei with $N \geqslant 52$ are rather small. The strength $\kappa$ of the quadrupole-quadrupole interaction is shown in panels (c) and (d). It exhibits a gradual decrease when moving away from the shell closure, a trend already found in previous IBM studies [47,53]. Note that around $N=40$ the strength $\kappa$ is much less sensitive to the neutron number than the parameter $\epsilon$. The parameter $\chi$ determines whether a nucleus is prolate $(\chi<0)$, oblate $(\chi>0)$, or $\gamma$-soft $(\chi \approx 0)$. As can be seen from panels (e) and (f), for the normal configuration in $\mathrm{Ge}$ nuclei, it changes sign from $N=38$ to 44 , which is consistent with the oblate-to-prolate transition observed for the minimum of the Gogny-D1M and mapped energy surfaces.

The strength of the three-body boson term also reflects $\gamma$-softness. In particular, a negative value of $\kappa^{\prime}$ creates a stable triaxial minimum at $\gamma=30^{\circ}$ whereas a positive value leads to stiffness along the $\gamma$ direction [see Eq. (9)]. From panels $(\mathrm{g})$ and $(\mathrm{h})$ one realizes that, for several of the considered $\mathrm{Ge}$ isotopes, the $\kappa^{\prime}$ values for the normal configurations are negative and notably large in magnitude. This reflects that the Gogny-D1M energy surfaces are generally $\gamma$-softer for Ge than for Se nuclei. The mixing strength $\omega$ [panels (i) and (j)] and the energy offset $\Delta$ [panels (k) and (1)] are of the same order of magnitude as those obtained in previous IBM configuration mixing calculations [15]. Note that the $\omega$ values are particularly large for $N=38$ in both the $\mathrm{Ge}$ and Se isotopic chains. In this case, the two minima observed in the Gogny-D1M energy surface are rather well separated from each other along the $\gamma$ direction and therefore large $\omega$ values are required.

\section{RESULTS FOR SPECTROSCOPIC PROPERTIES}

\section{A. Systematics of the excitation energies}

The excitation energies of the $2_{1}^{+}, 4_{1}^{+}, 0_{2}^{+}$and $2_{2}^{+}$states obtained in this work are displayed in Fig. 6 as functions of the neutron number. They are compared with the available experimental data [58]. As can be seen, our calculations provide a reasonable agreement with the experimental systematics, especially for the yrast states. The $E\left(2_{1}^{+}\right)$energy [panels (a) and (e)] can be regarded as one of the best signatures 

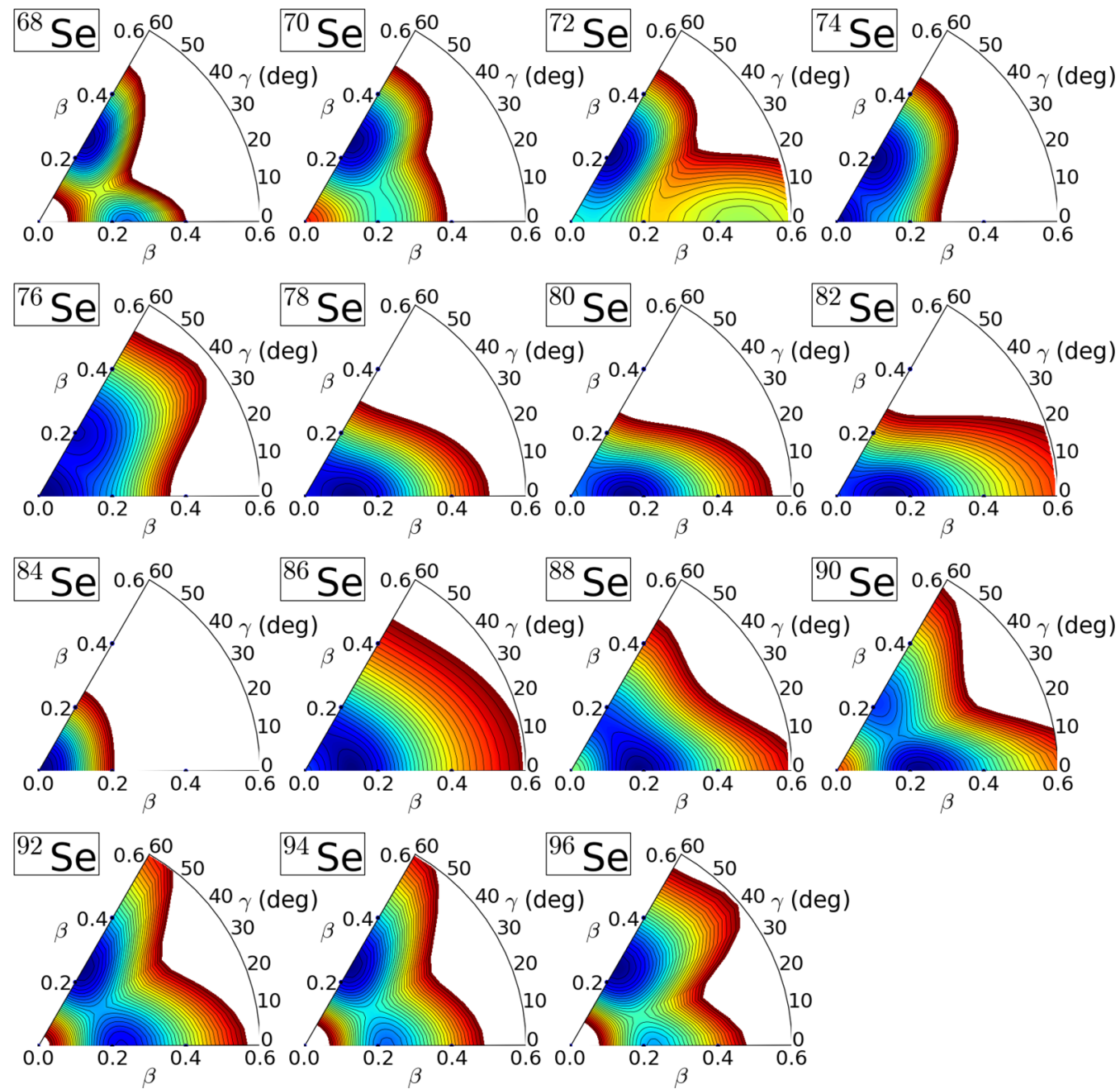

FIG. 4. The same as in Fig. 2, but for the mapped IBM energy surfaces.

for a shape/phase transition [3]. For both Ge and Se nuclei, the computed $E\left(2_{1}^{+}\right)$energies decrease as one approaches $N=40$. In the case of Ge isotopes, this is at variance with the experiment. This discrepancy could be attributed to the $N=40$ neutron subshell closure not explicitly taken into account in our calculations. Moreover, the $E\left(2_{1}^{+}\right)$values exhibit a pronounced peak at $N=50$. In the case of the $E\left(4_{1}^{+}\right)$ excitation energies [panels (b) and (f)], our results overestimate the experimental ones around $N=50$. This could be linked to the limited IBM configuration space comprising only $s$ and $d$ bosons. The inclusion of the $J=4^{+}(G)$ pair in the IBM model could improve the agreement with the experiment but lies out of the scope of this study. Work along these lines is in progress and will be reported elsewhere.

The appearance of low-lying $0_{2}^{+}$states is often attributed to intruder excitations and regarded as a signature of shape coexistence [17]. The predicted $E\left(0_{2}^{+}\right)$energies are plotted in panels (c) and (g). They display a pronounced decrease towards $N \approx 40$. This correlates well with the shape coexistence observed in the underlying Gogny-D1M energy surfaces around this neutron number. The overestimation of the $E\left(0_{2}^{+}\right)$ energy in the case of ${ }^{68} \mathrm{Ge}$ is due to the fact that a configuration mixing calculation has not been carried out in this case. The fraction of the intruder configuration in the IBM $0_{1}^{+}$and $0_{2}^{+}$ wave functions for $\mathrm{Ge}$ and Se nuclei is plotted in panels (a) and (b) of Fig. 7 as a function of the neutron number $N$. From the plots, one realizes that, for both $\mathrm{Ge}$ and $\mathrm{Se}$, the $0_{1}^{+}$and $\mathrm{O}_{2}^{+}$ states at $N=38$ mainly arise from the normal and intruder configuration, respectively. At $N=40$ and 42, in contrast, the $0_{1}^{+}$state is dominated by the intruder configuration, while the $\mathrm{O}_{2}^{+}$state is almost purely made of the normal configuration. Coming back to Fig. 6 [panels (c) and (g)], for the considered neutron-rich nuclei, several examples of low-lying $0_{2}^{+}$states are found beyond the $N=50$ shell closure. Finally, from the plots in panels (d) and (h), we conclude that our calculations lead to a reasonable description of the energies of the $2_{2}^{+}$states which are either interpreted as bandheads of the quasi- $\gamma$ bands or as members of the $0_{2}^{+}$bands.

We note, in both $\mathrm{Ge}$ and Se isotopes, that the predicted excitation energies of the non-yrast states $E\left(0_{2}^{+}\right)$and $E\left(2_{2}^{+}\right)$ are generally higher than the experimental values especially for $46 \leqslant N \leqslant 50$. This discrepancy has been commonly observed in our previous calculations for other mass regions using the HFB-to-IBM mapping procedure (see, e.g., Ref. [36]) and could be, in most cases, attributed to the restricted model space of the IBM when the shell closure is approached. 


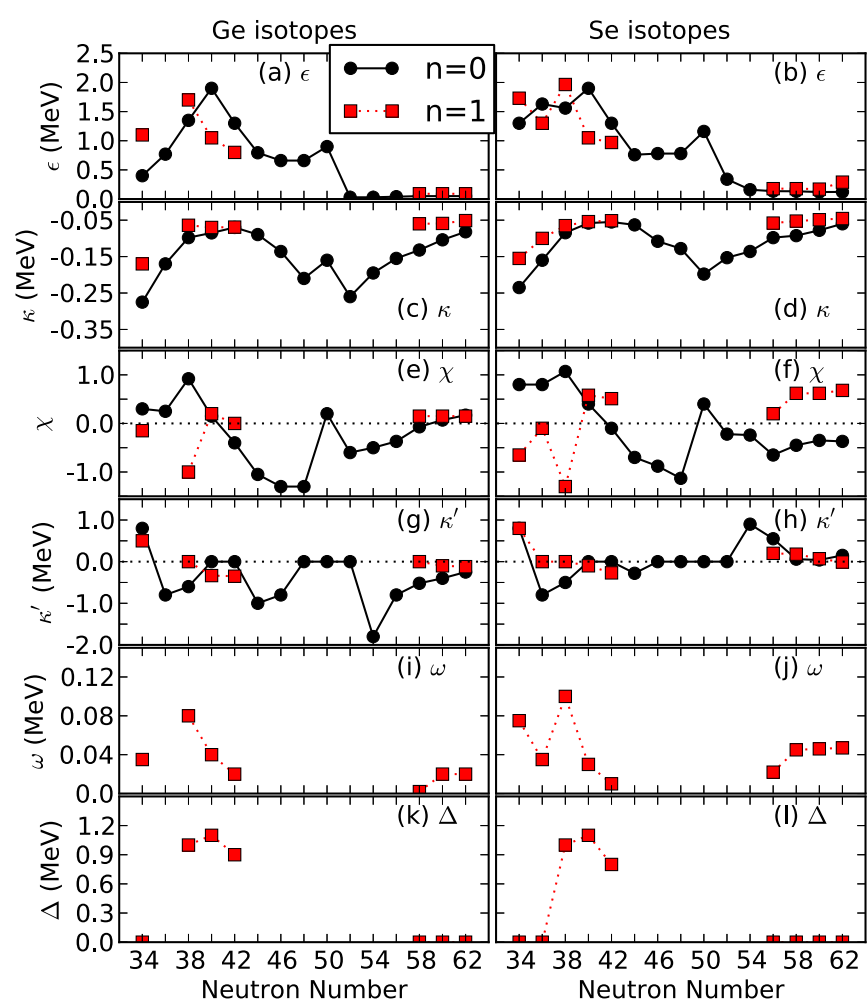

FIG. 5. The IBM parameters $\epsilon, \kappa, \chi, \kappa^{\prime}, \omega$, and $\Delta$ are depicted as functions of the neutron number, for the $[n=0]$ and $[n=1]$ configurations. For more details, see the main text.

\section{B. Electromagnetic properties}

\section{B(E2) transition rates}

The transition probabilities $B\left(E 2 ; 2_{1}^{+} \rightarrow 0_{1}^{+}\right), B\left(E 2 ; 4_{1}^{+}\right.$ $\left.\rightarrow 2_{1}^{+}\right), B\left(E 2 ; 0_{2}^{+} \rightarrow 2_{1}^{+}\right)$and $B\left(E 2 ; 2_{2}^{+} \rightarrow 2_{1}^{+}\right)$are depicted in Figs. 8 and 9 for $\mathrm{Ge}$ and $\mathrm{Se}$ nuclei, respectively. The maximum $B\left(E 2 ; 2_{1}^{+} \rightarrow 0_{1}^{+}\right)$value is reached around $N=40$, where the deformation is the largest [panel (a) of Figs. 8 and 9]. The agreement between our results and the experimental data for Ge and Se nuclei is fairly good. A similar trend is also found for the $B\left(E 2 ; 4_{1}^{+} \rightarrow 2_{1}^{+}\right)$transition rates [panel (b) of Figs. 8 and 9]. The quantity $B\left(E 2 ; 0_{2}^{+} \rightarrow 2_{1}^{+}\right)$, shown in panel (c) of Figs. 8 and 9 , can be regarded as a measure of the mixing between different intrinsic configurations. The experimental $B\left(E 2 ; 0_{2}^{+} \rightarrow 2_{1}^{+}\right)$value is very large around $N=38$ or 40 , where a pronounced configuration mixing could be expected. Such a large value is not reproduced in our calculations. In this case, the origin of the discrepancy between our predictions and the experimental results could be associated with a weak mixing between the $2_{1}^{+}$and $0_{2}^{+}$ states in our model. For both the Ge and Se chains, there are some discrepancies between the predicted and experimental $B\left(E 2 ; 2_{2}^{+} \rightarrow 2_{1}^{+}\right)$values at the quantitative level [panel (d) of Figs. 8 and 9]. Nevertheless, the experimental trend-i.e., the $B\left(E 2 ; 2_{2}^{+} \rightarrow 2_{1}^{+}\right)$transition probability reaching its largest value at around $N=40$, and being almost of the same order of magnitude as $B\left(E 2 ; 2_{1}^{+} \rightarrow 0_{1}^{+}\right)$-is reproduced rather well by the present calculation. Furthermore, the neutron number

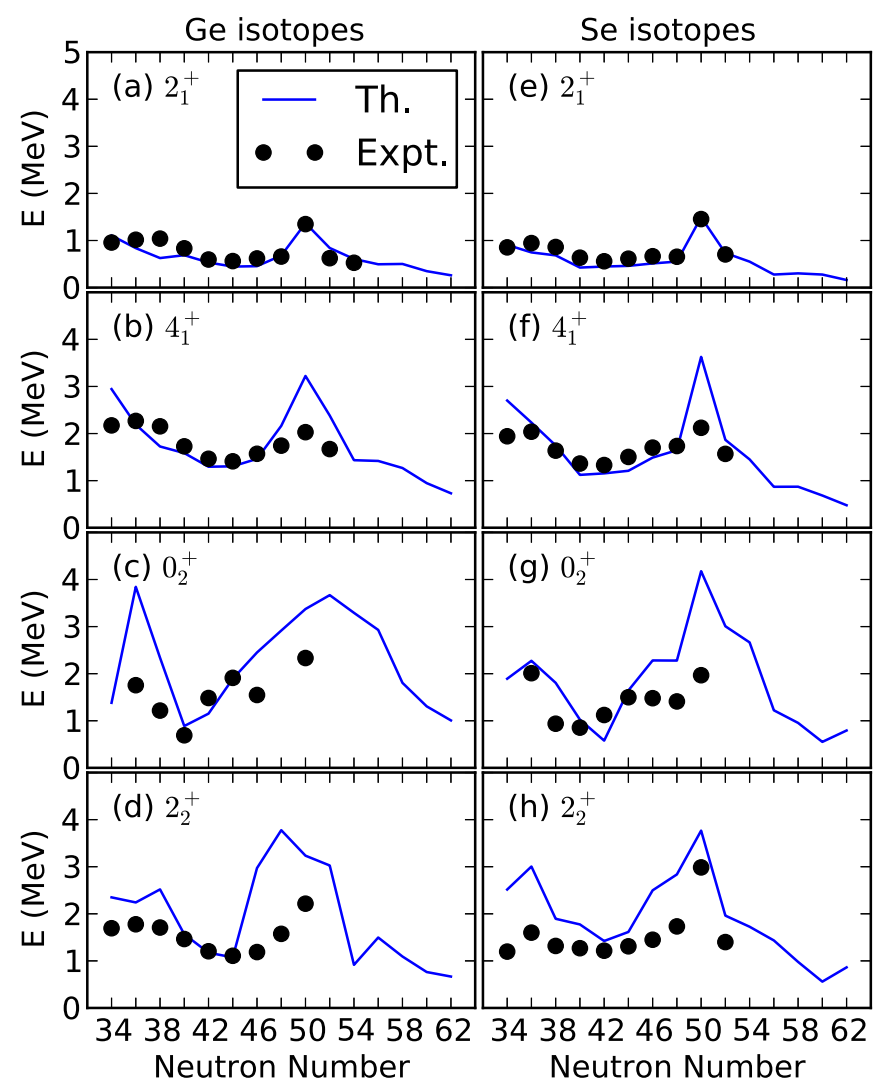

FIG. 6. The $2_{1}^{+}, 4_{1}^{+}, 0_{2}^{+}$, and $2_{2}^{+}$excitation energies obtained in the diagonalization of the mapped IBM Hamiltonian are plotted as functions of the neutron number, for the $\mathrm{Ge}$ and Se nuclei, along with the available experimental data [58].

$N=40$ is precisely the region where the Gogny-D1M energy surfaces display a pronounced $\gamma$-softness.

\section{Spectroscopic quadrupole moments}

The spectroscopic quadrupole moments $Q_{s p}$ corresponding to the $2_{1}^{+}$and $2_{2}^{+}$states in Ge and Se nuclei, are shown in Fig. 10 where, they are also compared with the available experimental

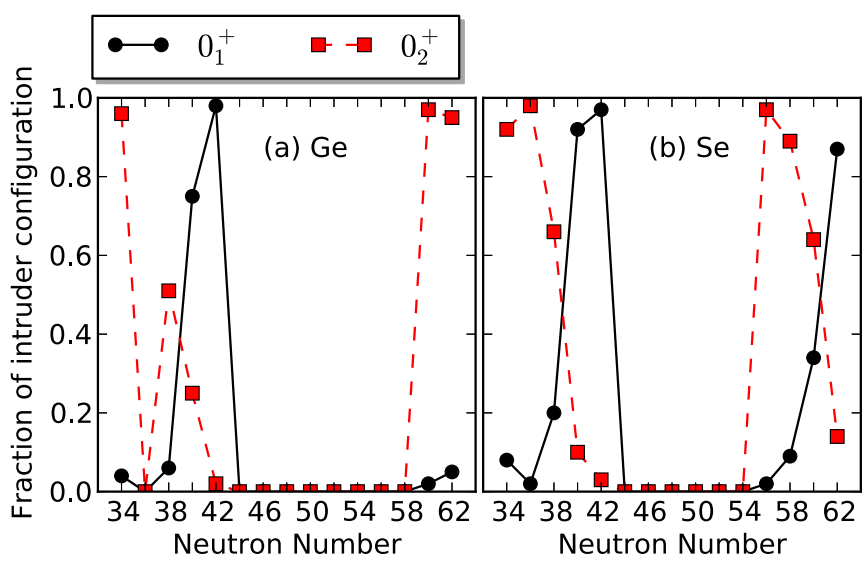

FIG. 7. Fraction of the intruder configuration in the IBM $0_{1}^{+}$and $\mathrm{O}_{2}^{+}$wave functions. For more details, see the main text. 


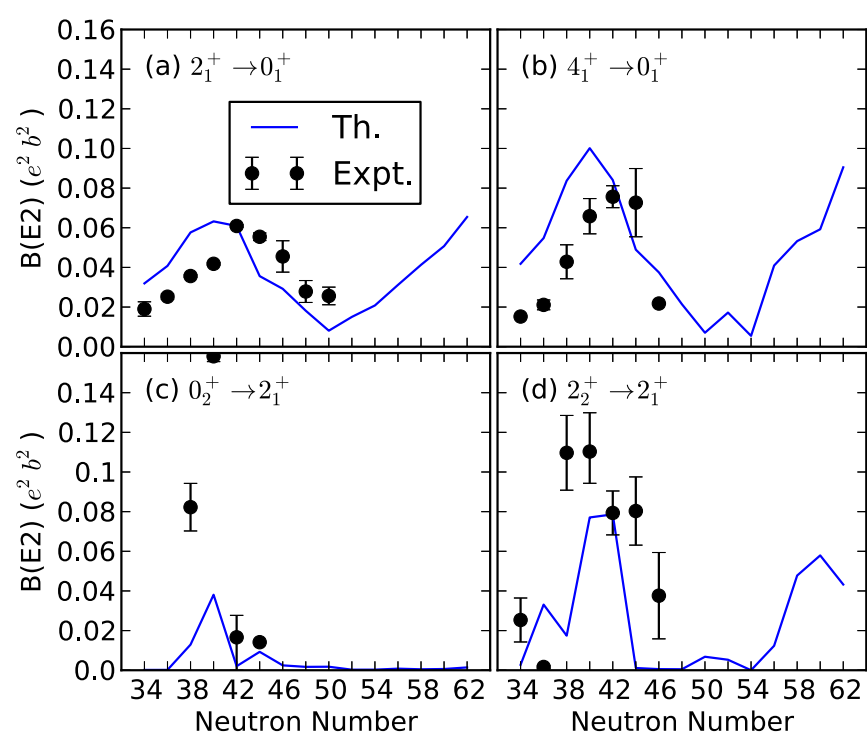

FIG. 8. The $B\left(E 2 ; 2_{1}^{+} \rightarrow 0_{1}^{+}\right), B\left(E 2 ; 4_{1}^{+} \rightarrow 2_{1}^{+}\right), B\left(E 2 ; 0_{2}^{+} \rightarrow\right.$ $\left.2_{1}^{+}\right)$, and $B\left(E 2 ; 2_{2}^{+} \rightarrow 2_{1}^{+}\right)$transition probabilities obtained for $\mathrm{Ge}$ isotopes are plotted as functions of the neutron number. Experimental data have been taken from Ref. [58].

data $[58,61]$. The predicted postive spectroscopic quadrupole moments $Q_{s p}\left(2_{1}^{+}\right)$for $34 \leqslant N \leqslant 38$ [panel (a)] indicate that the corresponding states are oblate. In our calculations, the $2_{1}^{+}$wave functions for ${ }^{72,74} \mathrm{Ge}$ are dominated by the intruder oblate and triaxial configurations, respectively. Consequently, their $Q_{s p}\left(2_{1}^{+}\right)$moments are positive and nearly zero, whereas experimentally $Q_{s p}\left(2_{1}^{+}\right)<0$ at both $N=40$ and 42 . Similarly, at variance with the data, the predicted $Q_{s p}\left(2_{2}^{+}\right)$are negative and approximately 0 for ${ }^{72} \mathrm{Ge}$ and ${ }^{74} \mathrm{Ge}$, respectively. With the exception of ${ }^{82} \mathrm{Ge}$ for which $Q_{s p}\left(2_{1}^{+}\right) \approx 0$, the predicted spectroscopic quadrupole moments $Q_{s p}\left(2_{1}^{+}\right)$are negative for most of the heavier Ge isotopes. The sign of the $Q_{s p}\left(2_{2}^{+}\right)$

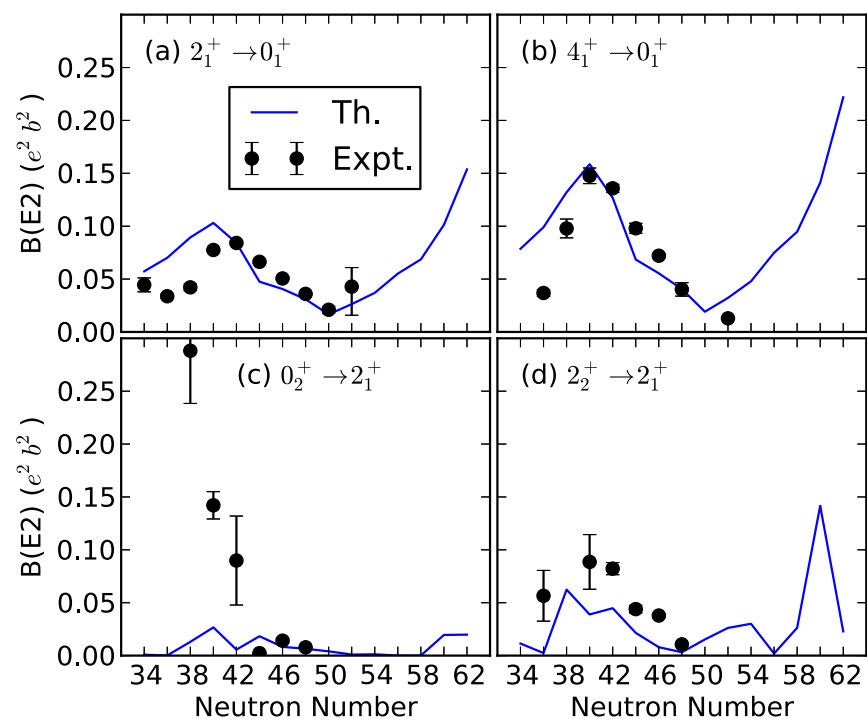

FIG. 9. The same as in Fig. 8, but for the Se isotopes. Experimental data have been taken from Ref. [58].

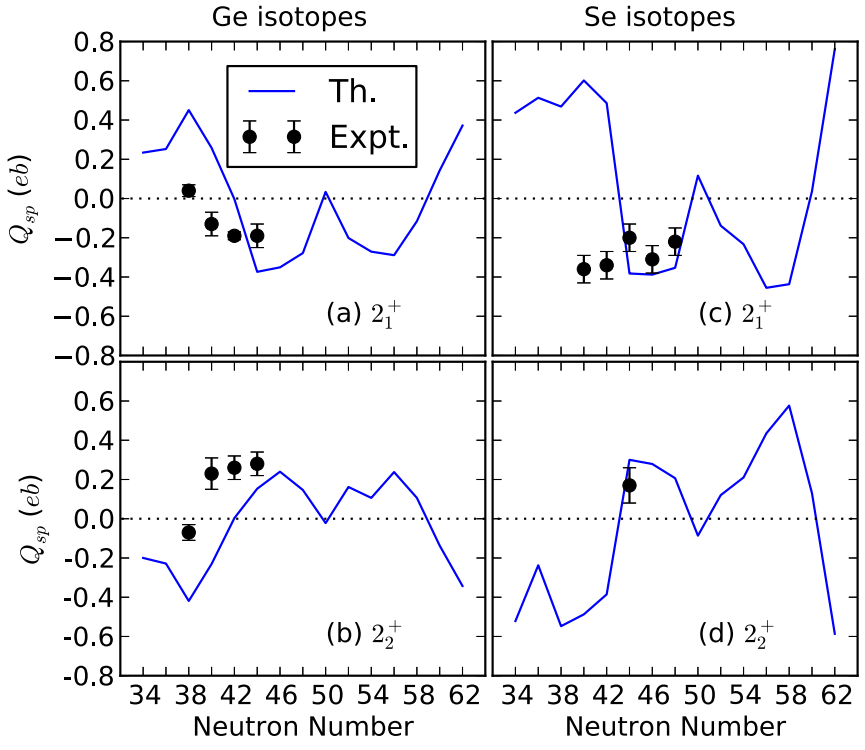

FIG. 10. Spectroscopic quadrupole moments $Q_{s p}$ for the $2_{1}^{+}$ and $2_{2}^{+}$states in $e \mathrm{~b}$ units. The experimental values are taken from Refs. [58,61].

values [panel (b)] is the opposite to the one of the $Q_{s p}\left(2_{1}^{+}\right)$ moments. As can be seen from the figure [panels (a) and (b)] our calculations qualitatively follow the experimental trends for both $Q_{s p}\left(2_{1}^{+}\right)$and $Q_{s p}\left(2_{2}^{+}\right)$in $\mathrm{Ge}$ isotopes, i.e., the decrease [increase] in $Q_{s p}\left(2_{1}^{+}\right)\left[Q_{s p}\left(2_{2}^{+}\right)\right]$as a function of $N$ from $N=38$ to 44 . Similar conclusions can be drawn for the predicted $Q_{s p}$ moments in the case of Se isotopes [panels (c) and (d)]. In particular, our results for $Q_{s p}\left(2_{1}^{+}\right)$agree well with the experimental ones at $N=44,46$, and 48 as well as with the only available data on $Q_{s p}\left(2_{2}^{+}\right)$at $N=44$. Nevertheless, at variance with the experiment, in our calculations $Q_{s p}\left(2_{1}^{+}\right)>0$ for ${ }^{74,76} \mathrm{Se}$, similarly to their isotones ${ }^{72,74} \mathrm{Ge}$.

\section{E0 properties}

The $E 0$ transition strength values between $0^{+}$states can be regarded as a signature of both shape/phase transitions and shape coexistence. The $\rho^{2}\left(E 0 ; 0_{2}^{+} \rightarrow 0_{1}^{+}\right)$values, obtained within the mapped IBM framework, for Ge and Se nuclei are compared with the available experimental data [59] in Fig. 11. The peaks observed in the predicted $\rho^{2}\left(E 0 ; 0_{2}^{+} \rightarrow 0_{1}^{+}\right)$values, shown in panels (a) and (b) of the figure, characterize the structural evolution along both isotopic chains. In the case of the Ge isotopes, for example, the peak at $N \approx 38$ can be associated with the emergence of shape coexistence while the increase in the predicted $\rho^{2}\left(E 0 ; 0_{2}^{+} \rightarrow 0_{1}^{+}\right)$values towards $N=44$ suggests the development of quadrupole collectivity in the considered nuclei. For both chains $\rho^{2}\left(E 0 ; 0_{2}^{+} \rightarrow 0_{1}^{+}\right) \approx 0$ at $N=42$, considerably underestimating the experimental value. This implies that the mixing between the $0_{1}^{+}$and $0_{2}^{+}$ states is too weak in this case. As already shown in Fig. 7, for ${ }^{74} \mathrm{Ge}$ and ${ }^{76} \mathrm{Se}$, the $0_{1}^{+}$state in the present analysis is made almost entirely of the intruder $(\gamma$-soft) configuration while the intruder component is negligible in the $0_{2}^{+}$state. As can be seen from panel (b), $\rho^{2}\left(E 0 ; 0_{2}^{+} \rightarrow 0_{1}^{+}\right)$becomes larger 


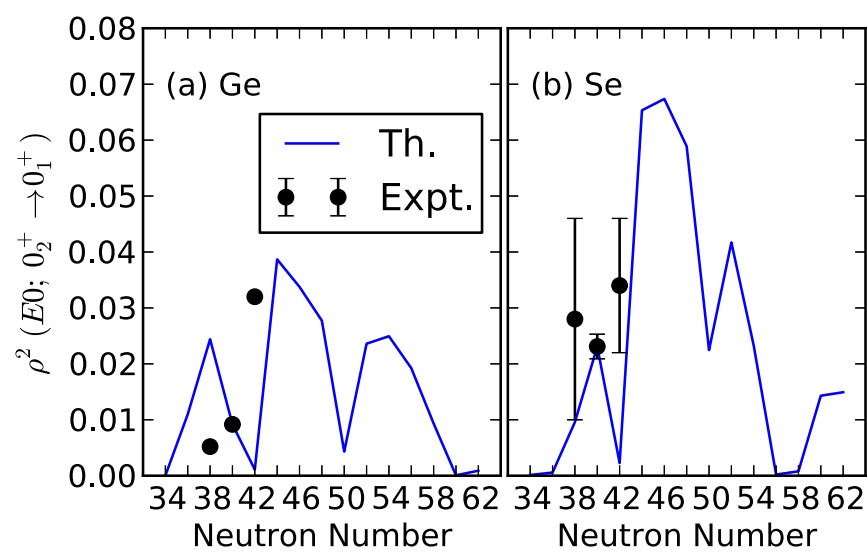

FIG. 11. The $\rho^{2}\left(E 0 ; 0_{2}^{+} \rightarrow 0_{1}^{+}\right)$values, obtained within the mapped IBM framework for Ge and Se nuclei, are compared with the available experimental data taken from Ref. [59].

for neutron-rich Se isotopes with $N \geqslant 60$. This indicates the strong mixing between the normal and intruder configurations in their $0_{1}^{+}$and $0_{2}^{+}$wave functions [see panel (b) of Fig. 7].

\section{Level schemes of selected isotopes}

In this section, we further demonstrate the ability of our fermion-to-boson mapping procedure to describe not only the overall systematics of the spectroscopic properties in the studied Ge and Se chains but also to account for the detailed band structures and decay patterns of individual nuclei in comparison with the experiment. In particular, we consider the nuclei ${ }^{70,72,74} \mathrm{Ge}$ and ${ }^{72,74,76} \mathrm{Se}$ which correspond to an abrupt shape transition and the emergence of shape coexistence in their isotopic chains. We will also discuss the level schemes obtained for the $N=60$ isotones ${ }^{92} \mathrm{Ge}$ and ${ }^{94} \mathrm{Se}$. The level schemes presented in what follows have been classified into bands according to their dominant $E 2$ decays.

\section{1. $N=38$ isotones}

The low-energy level schemes obtained for the $N=38$ isotones ${ }^{70} \mathrm{Ge}$ and ${ }^{72} \mathrm{Se}$ are depicted in Figs. 12 and 13. The experimental ground-state band exhibits an almost equal spacing between its members. On the other hand, the theoretical ground-state band, mainly coming from the oblate normal configuration (see Fig. 7), rather looks like a regular collective band approximately following the $J(J+1)$ systematics in the rotational limit, and is more stretched for higher spins. This could be due to the fact that the Gogny-D1M energy surfaces for these nuclei exhibit a rather pronounced oblate minimum (see Figs. 1 and 2), and the resultant mapped Hamiltonian gives a more collective feature than is suggested experimentally. In the case of ${ }^{70} \mathrm{Ge}$, our calculations provide the band built on the $0_{2}^{+}$state (almost $50 \%$ of the wave function is made of the intruder prolate configuration) as well as the quasi- $\gamma$ band with the sequence of states $2^{+}, 3^{+}, 4^{+}, 5^{+}, 6^{+}, \ldots$. The bandheads of these bands are rather overestimated. Similar excited bands are found for ${ }^{72} \mathrm{Se}$, but the one built on the $0_{2}^{+}$state is lower than the quasi- $\gamma$ band.

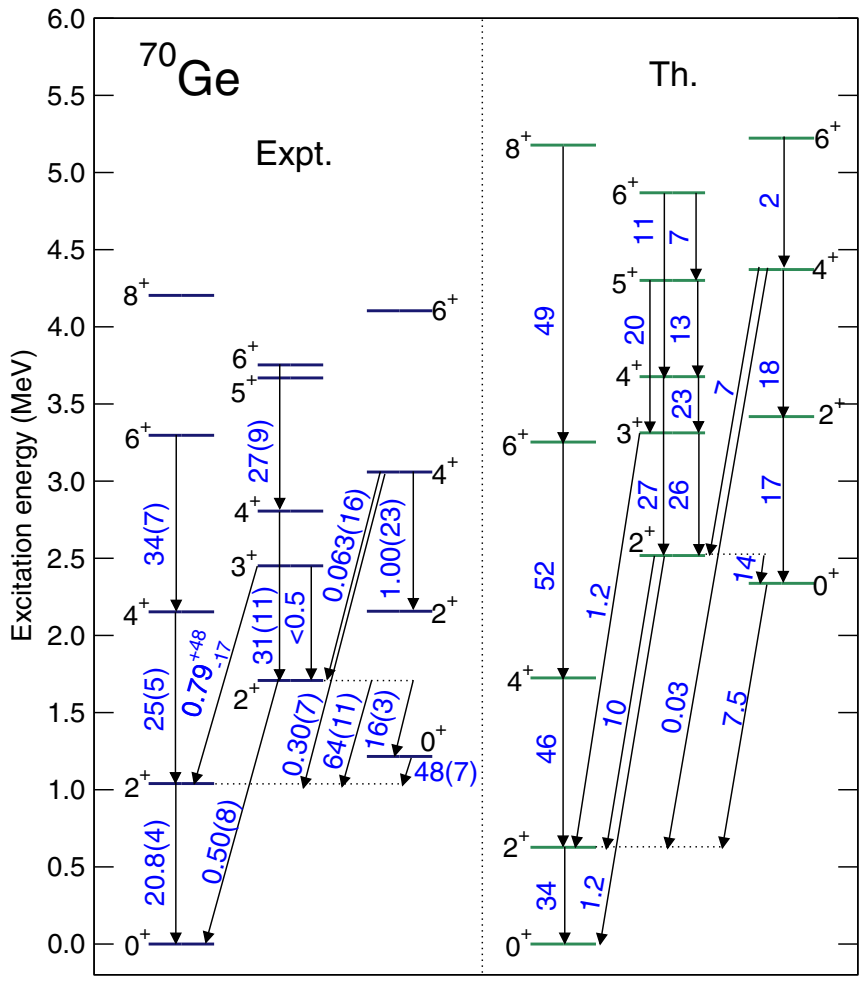

FIG. 12. Low-energy level scheme for ${ }^{70} \mathrm{Ge}$. The numbers (in blue) near the arrows stand for the $B(E 2)$ transition strengths in Weisskopf units. Experimental data have been taken from Ref. [58].

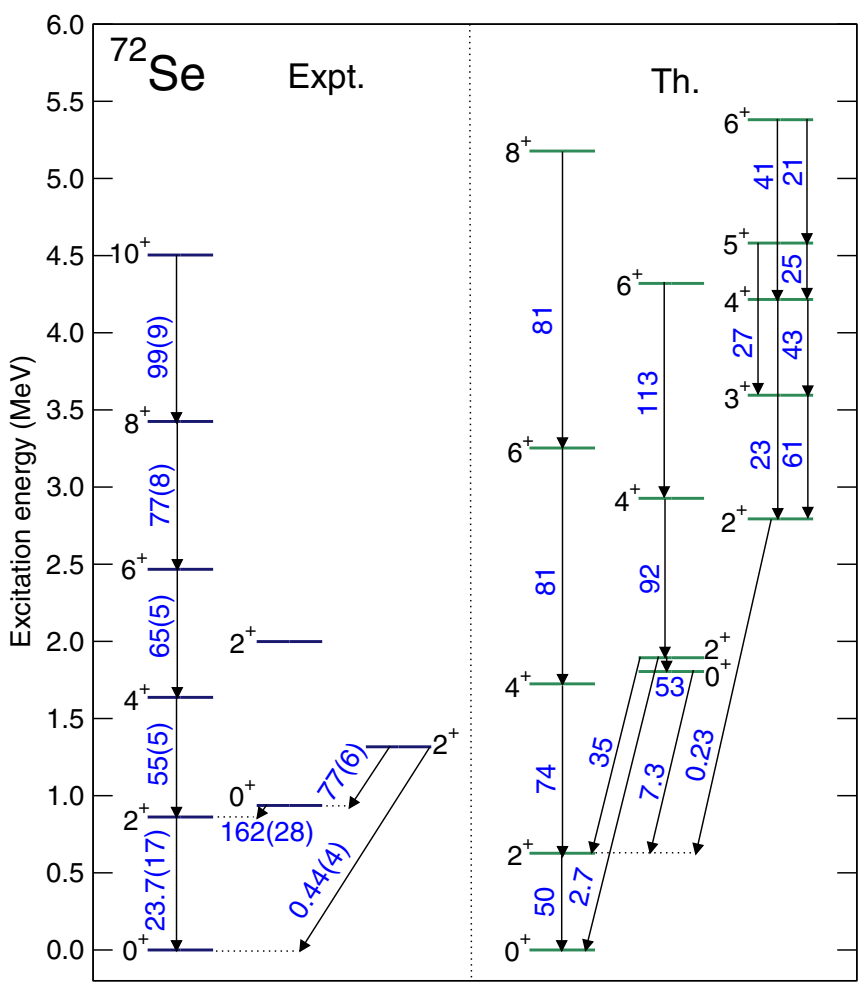

FIG. 13. The same as in Fig. 12, but for ${ }^{72} \mathrm{Se}$. 


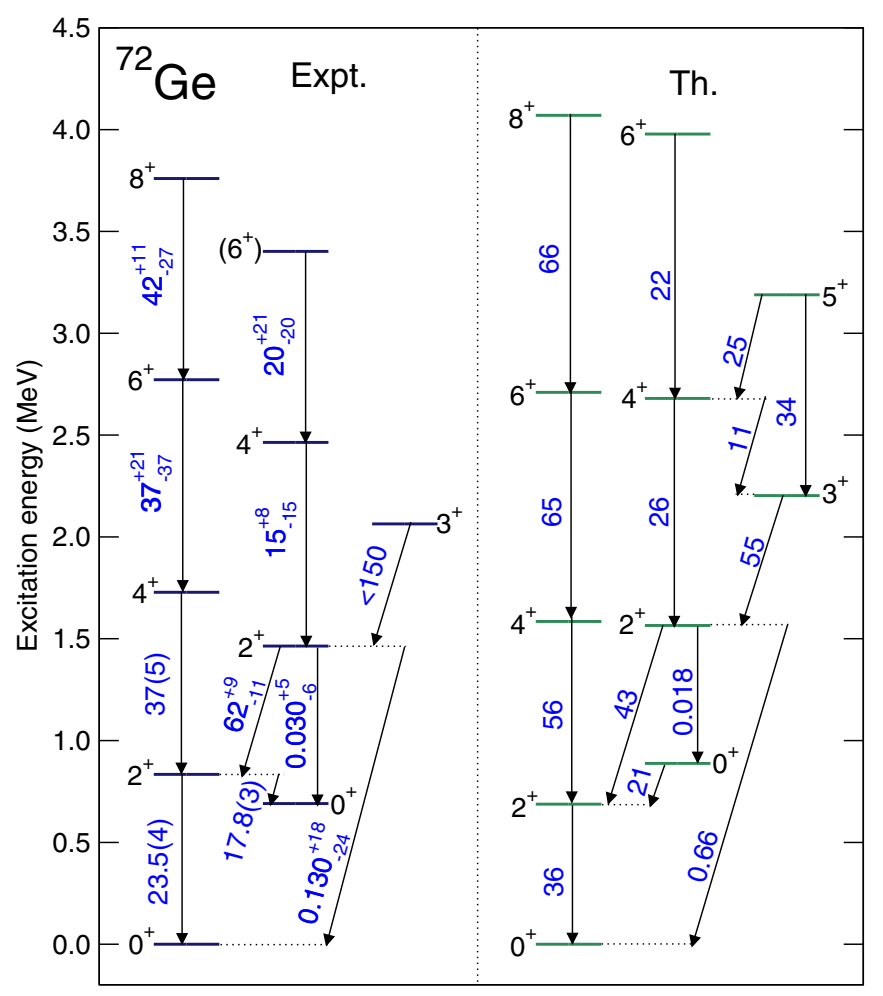

FIG. 14. The same as in Fig. 12, but for ${ }^{72} \mathrm{Ge}$.

\section{2. $N=40$ isotones}

The low-energy level schemes, obtained for the nuclei ${ }^{72} \mathrm{Ge}$ and ${ }^{74} \mathrm{Se}$, are compared in Figs. 14 and 15 with the experimental data. For those $N=40$ isotones, the corresponding Gogny-D1M energy surfaces exhibit a coexistence between spherical and oblate shapes. From the experimental point of view, the energy of the $0_{2}^{+}$state is the lowest precisely at $N=40$. This, together with the strong $B\left(E 2 ; 0_{2}^{+} \rightarrow 2_{1}^{+}\right)$ transition and the $Q_{s p}\left(2^{+}\right)$values shown in Fig. 10, suggest a pronounced mixing between oblate and prolate configurations in those nuclear systems. As can be seen from Figs. 14 and 15 , our calculations describe well the experimental spectra (including the energy of the $0_{2}^{+}$states) and $B(E 2)$ transition probabilities. However, as already discussed in Sec. VB, the $B\left(E 2 ; 0_{2}^{+} \rightarrow 2_{1}^{+}\right)$value is underestimated within our model because the mixing between the two configurations is not strong enough. Previous calculations, within the 5D collective Hamiltonian approach [13], have also provided a reasonable description of the low-energy spectra and decay patterns for the same nuclei while overestimating the $0_{2}^{+}$energy in ${ }^{72} \mathrm{Ge}$.

\section{3. $N=42$ isotones}

The spectra obtained for ${ }^{74} \mathrm{Ge}$ and ${ }^{76} \mathrm{Se}$ are compared, in Figs. 16 and 17, with the experimental ones. At $N=42$, our Gogny-D1M energy surfaces display a coexistence between spherical and $\gamma$-soft minima. From the experimental point of view, the lower energy of the $2_{2}^{+}$bandhead of the quasi- $\gamma$ band and the strong $B\left(E 2 ; 2_{2}^{+} \rightarrow 2_{1}^{+}\right)$transition suggest that those $N=42$ isotones could be considered as examples of $\gamma$-softness. Our calculations describe reasonably well the

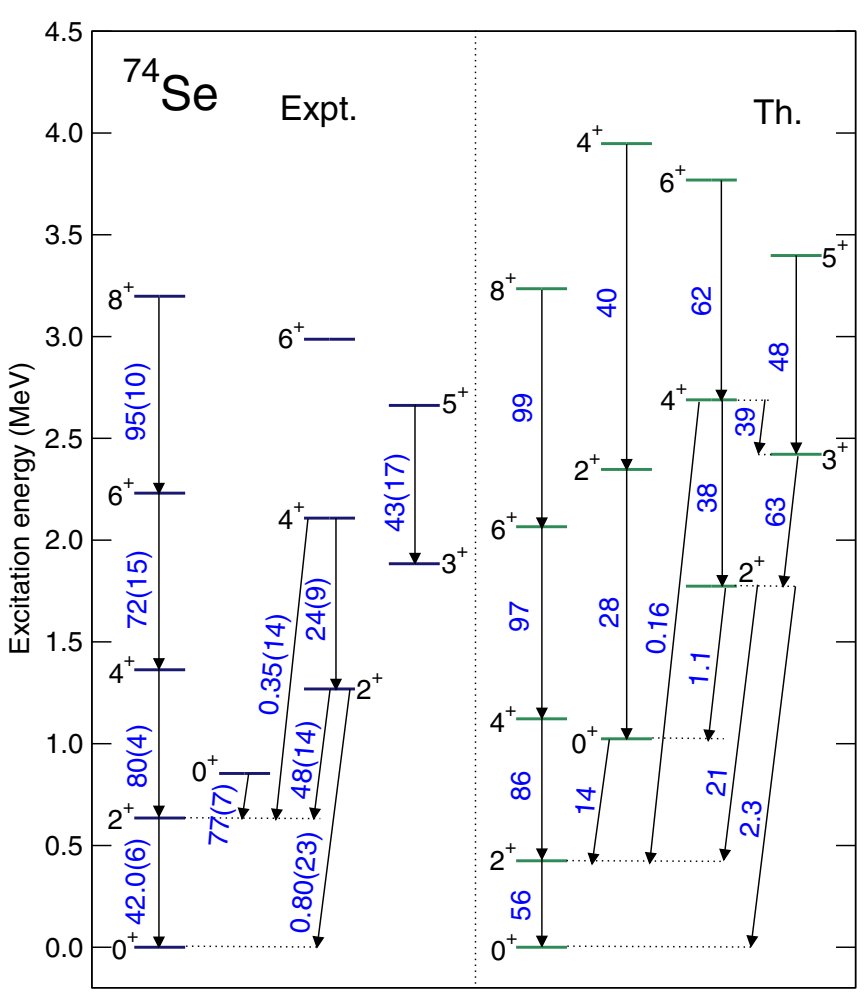

FIG. 15. The same as in Fig. 12, but for ${ }^{74} \mathrm{Se}$.

quasi- $\gamma$ band with the sequence of states $2_{\gamma}^{+}, 3_{\gamma}^{+}, 4_{\gamma}^{+}, 5_{\gamma}^{+}, \ldots$ as well as the $B\left(E 2 ; 2_{2}^{+} \rightarrow 2_{1}^{+}\right)$transition probability, which is comparatively as large as the $B\left(E 2 ; 2_{1}^{+} \rightarrow 0_{1}^{+}\right)$value. In the case of ${ }^{76} \mathrm{Se}$, both the computed and empirical quasi- $\gamma$ bands consist of $3_{\gamma}^{+}$and $4_{\gamma}^{+}$levels close in energy following the systematics of the $\gamma$-unstable-rotor model of Wilets and Jean [62]. Furthermore, the ground-state bands build on the $0_{1}^{+}$ states obtained for ${ }^{74} \mathrm{Ge}$ and ${ }^{76} \mathrm{Se}$ are largely made of the intruder configurations corresponding to triaxial $\left(\gamma \approx 30^{\circ}\right)$ and oblate $\left(\gamma=60^{\circ}\right)$ minima in the Gogny-D1M energy surfaces, respectively. On the other hand, the $0_{2}^{+}$energy for ${ }^{76} \mathrm{Se}$ is underestimated in the present calculation. Another deviation of the predicted level scheme from the experimental one for ${ }^{76} \mathrm{Se}$ is the fact that the $B(E 2 ; J \rightarrow J-2)\left(J=2^{+}, 4^{+}, 6^{+}, 8^{+}\right)$ transition strength in the predicted yrast band increases with spin but drops suddenly at $J=8^{+}$, while experimentally no such sudden decrease is observed, even considering the experimental uncertainty. This reflects a general feature of the IBM [34]: due to the finite boson number the in-band E2 transition strength increases with spin, then reaches its maximum value at certain spin, and finally decreases. For both ${ }^{74} \mathrm{Ge}$ and ${ }^{76} \mathrm{Se}$, the $B\left(E 2 ; 0_{2}^{+} \rightarrow 2_{1}^{+}\right)$transition probability is largely underestimated in the calculation. We remind the reader also that the predicted $\rho^{2}\left(E 0 ; 0_{2}^{+} \rightarrow 0_{1}^{+}\right)$values for these $N=42$ isotones are too small compared to the experimental values [see Figs. 11(a) and 11(b)]. Both of these discrepancies have the same origin as the ones in the case of the $N=40$ isotones. 


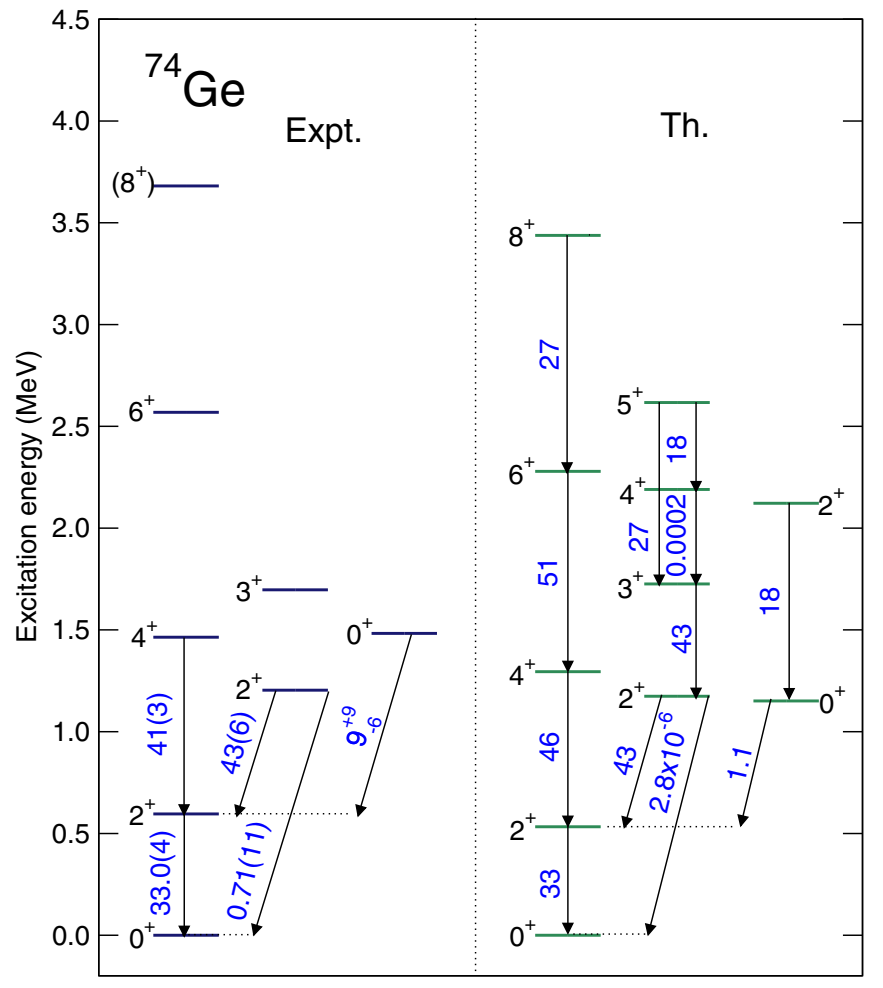

FIG. 16. The same as in Fig. 12, but for ${ }^{74} \mathrm{Ge}$.

\section{4. $N=60$ isotones}

The spectra obtained for the neutron-rich nuclei ${ }^{92} \mathrm{Ge}$ and ${ }^{94} \mathrm{Se}$ are shown in Fig. 18. Within the Gogny-D1M HFB framework, a $\gamma$-soft oblate minimum has been found for the former while for the latter our calculations predict prolate-oblate shape coexistence. The first excited band for ${ }^{92} \mathrm{Ge}$ is predicted to be a quasi- $\gamma$ band. The large $B\left(E 2 ; 2_{2}^{+} \rightarrow\right.$ $2_{1}^{+}$) value obtained for this nucleus (of the same order of magnitude as the in-band $B\left(E 2 ; 2_{1}^{+} \rightarrow 0_{1}^{+}\right)$strength) indicates a pronounced $\gamma$-softness. Furthermore, the $2^{+}$and $3^{+}$as well as the $4^{+}$and $5^{+}$levels of the quasi- $\gamma$ band are close to each other, which is rather consistent with the rigid-triaxial-rotor picture of Davydov and Filippov [63]. In the case of ${ }^{94} \mathrm{Se}$, the level scheme suggests two coexisting $0^{+}$bands (the $0_{1}^{+}$groundstate band coming from the oblate normal configuration and the $0_{2}^{+}$band coming from the prolate intruder configuration). The small $B\left(E 2 ; 0_{2}^{+} \rightarrow 2_{1}^{+}\right)$transition probability confirms the weak mixing between oblate and prolate configurations in this nucleus. Our calculations also provide a quasi- $\gamma$ band that exhibits the rigid-triaxial-rotor feature, being much higher in energy than in ${ }^{92} \mathrm{Ge}$.

\section{SENSITIVITY ANALYSIS}

As already pointed out in previous sections, there are several model assumptions that could affect our results for the spectroscopic properties of the studied nuclei. In this section, we turn our attention to the sensitivity of our results with respect to the underlying Gogny EDF that provides the starting point for our fermion-to-boson mapping scheme. To this end,

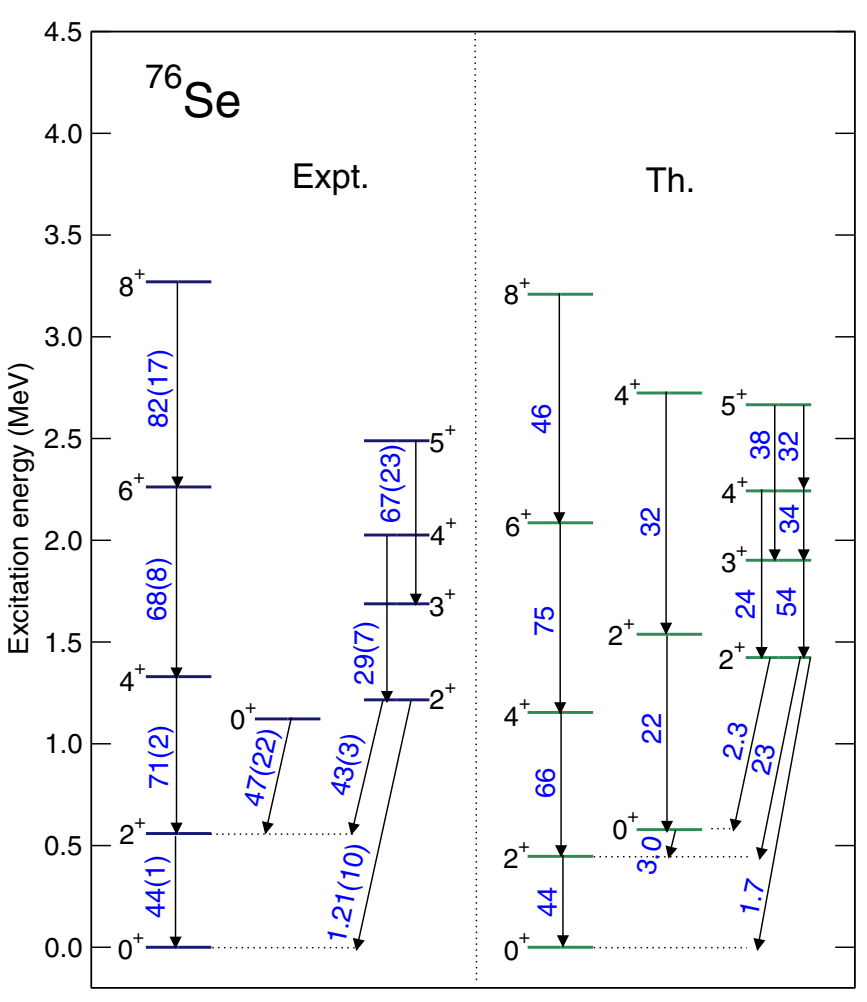

FIG. 17. The same as in Fig. 12, but for ${ }^{76} \mathrm{Se}$.

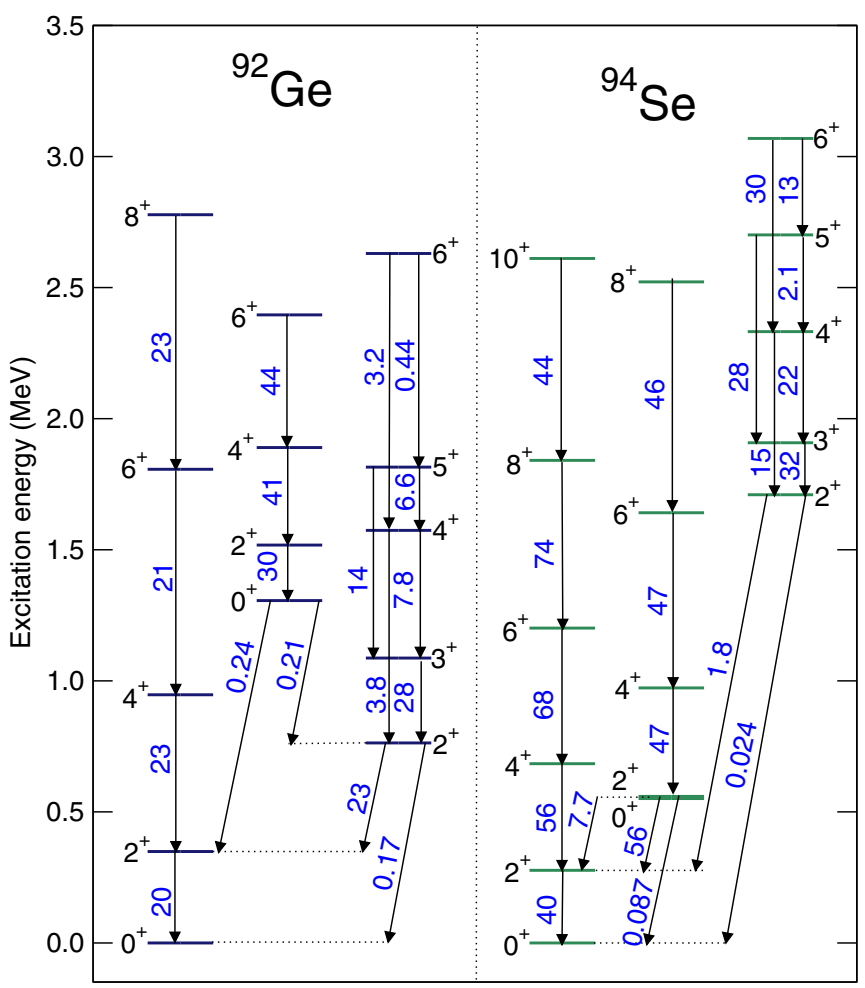

FIG. 18. Low-energy level schemes for the $N=60$ isotones ${ }^{92} \mathrm{Ge}$ and ${ }^{94} \mathrm{Se}$. Note that the theoretical $\mathrm{O}_{2}^{+}$and $2_{2}^{+}$levels of ${ }^{94} \mathrm{Se}$ are almost degenerate. The $B(E 2)$ transition strengths relevant to these states are $B\left(E 2 ; 0_{2}^{+} \rightarrow 2_{1}^{+}\right)=7.7, B\left(E 2 ; 2_{2}^{+} \rightarrow\right.$ $\left.2_{1}^{+}\right)=56, B\left(E 2 ; 2_{2}^{+} \rightarrow 0_{1}^{+}\right)=0.087, B\left(E 2 ; 2_{2}^{+} \rightarrow 0_{2}^{+}\right)=38$, and $B\left(E 2 ; 4_{2}^{+} \rightarrow 2_{2}^{+}\right)=47$ W.u. 

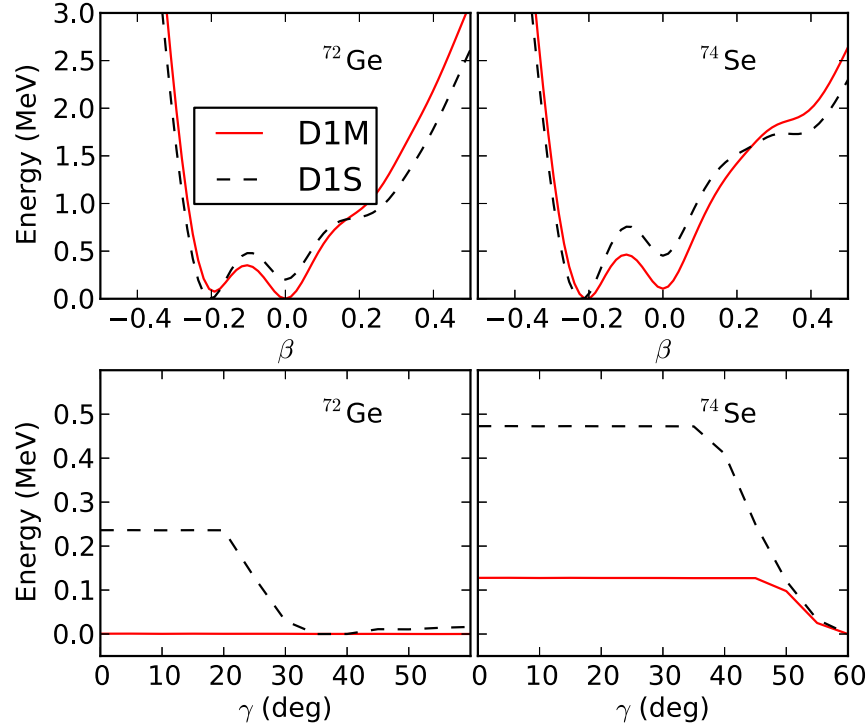

FIG. 19. The Gogny-HFB energy curves for the $N=40$ isotones ${ }^{72} \mathrm{Ge}$ and ${ }^{74} \mathrm{Se}$ are depicted (upper panels) as functions of the axial deformation parameter $\beta\left(\gamma=0^{\circ}\right)$. In the lower panels the HFB energies are shown as functions of $\gamma$. For each $\gamma$ value the parameter $\beta$ is chosen so as to minimize the energy. Results are shown for both the Gogny-D1S and Gogny-D1M EDFs.

in the upper panels of Fig. 19 we have plotted the Gogny-HFB energy curves for the $N=40$ isotones ${ }^{72} \mathrm{Ge}$ and ${ }^{74} \mathrm{Se}$ as functions of the axial deformation parameter $\beta\left(\gamma=0^{\circ}\right)$. In the lower panels of the same figure, we have depicted the HFB energies as functions of $\gamma$ taking for the parameter $\beta$ the value that minimizes the energy for each value of $\gamma$. At the quantitative level there are certain differences between the results provided by the two functionals. For example, the D1M energy curve for ${ }^{72} \mathrm{Ge}$ exhibits a global spherical minimum while an oblate one is obtained with the D1S parametrization. On the other hand, for ${ }^{74} \mathrm{Se}$, both parameter sets lead to an oblate global minimum but we obtain a softer behavior along the $\gamma$ direction with the Gogny-D1M than with the Gogny-D1S EDF (see lower panels).

The spectra obtained for ${ }^{72} \mathrm{Ge}$ and ${ }^{74} \mathrm{Se}$ with the two Gogny EDFs are compared in Fig. 20. The experimental data are also included in the plots to facilitate the comparison. It is satisfying to observe that there is no major difference between the spectra provided by both parametrizations of the GognyEDF, except for the $0_{2}^{+}$energy level. Such a difference could be attributed to the different topology of the corresponding HFB energy surfaces. We have also checked that the spectroscopic properties obtained for all the considered nuclei ${ }^{66-94} \mathrm{Ge}$ and ${ }^{68-96} \mathrm{Se}$ with the D1S parametrization are almost identical to the ones obtained with the D1M set. This is the reason why we have not discussed them in detail in the present paper.

\section{CONCLUSION}

In this study, we have considered both the shape/phase transitions and shape coexistence in the $\mathrm{Ge}$ and $\mathrm{Se}$ isotopic chains. To this end, calculations have been carried out for the

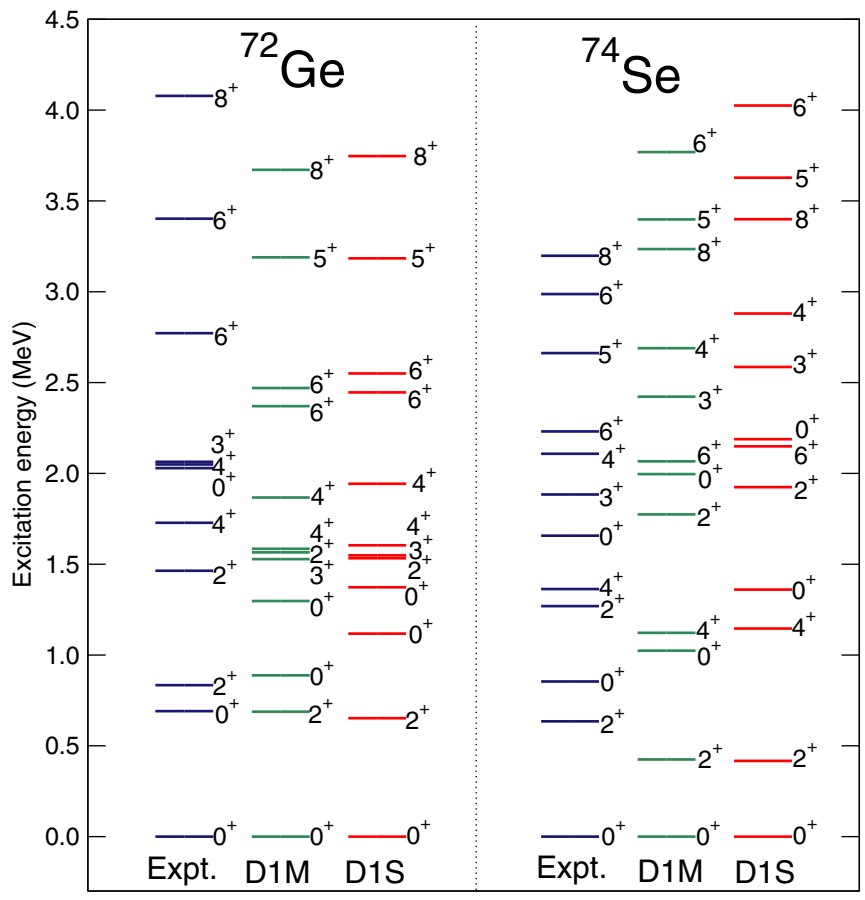

FIG. 20. Low-energy spectra obtained for ${ }^{72} \mathrm{Ge}$ and ${ }^{74} \mathrm{Se}$ within the fermion-to-boson mapping procedure based on the Gogny-D1S and Gogny-D1M EDFs. The experimental spectra are also included to facilitate the comparison.

nuclei ${ }^{66-94} \mathrm{Ge}$ and ${ }^{68-96}$ Se within the Gogny-HFB framework and, subsequently, within the mapped IBM approximation. The IBM configuration mixing Hamiltonian, with parameters determined through the mapping procedure, has been diagonalized, and the resulting wave functions have been used to compute the spectroscopic properties of the considered nuclei. Though a restricted form of the IBM-1 Hamiltonian has been employed, our calculations provide a reasonable description of the systematics for the low-lying energy spectra and transition strengths.

The Gogny-D1M energy surfaces predict the coexistence between the prolate and oblate shapes in the lightest nuclei in both isotopic chains. For shapes around $N=40$ coexistence between spherical and $\gamma$-soft shapes is observed. When neutron number increases towards the $N=50$ shell closure, weakly deformed prolate shapes are obtained. On the other hand, for $52 \leqslant N \leqslant 62$ a number of nuclei exhibiting $\gamma$-soft shapes and coexistence between prolate and oblate shapes are observed. The behaviors of the derived IBM parameters, resulting low-lying energy levels, $B(E 2)$ transition strengths, spectroscopic quadrupole moments, and $\rho^{2}\left(E 0 ; 0_{2}^{+} \rightarrow 0_{1}^{+}\right)$ values correlate well with the systematic of the Gogny-D1M energy surface. Through the analysis of the IBM wave functions, the low-lying $0_{2}^{+}$state around $N=40$ has been shown to arise either from the intruder configuration associated with the $\gamma$-soft minimum or the normal configuration associated with the closely lying spherical ground state minimum. Around this neutron number, our calculation also identifies signatures of $\gamma$ softness. On the neutron-rich side with $N \approx 60$, our 
calculation further predicts many examples of the $\gamma$-soft spectra and low-lying $0_{2}^{+}$band.

On the other hand, we have also pointed out several discrepancies between our results and experimental data. In particular, our calculation underestimates the $B\left(E 2 ; 0_{2}^{+} \rightarrow 2_{1}^{+}\right)$transition strength for $N \leqslant 42$, indicating that the mixing between the different configurations is too small. This is obviously due to the chosen parameters for the IBM Hamiltonian, particularly the too small strength parameter of the mixing interaction which could be a consequence of the topology of the Gogny EDF energy surfaces and/or the assumptions made at the IBM level. In this respect, the form of the IBM Hamiltonian employed in this study may be too simple, and some additional terms could be included in the Hamiltonian. As is well known, the use of the IBM-1 is particularly justified for heavy nuclei, where protons and neutrons occupy different major shells [47]. However, the lightest isotopes considered in this work have nearly equal $Z$ and $N$ values and, therefore, the presence of proton-neutron pairing effects might not be negligible. More realistic calculation should employ versions of the IBM that explicitly include isospin degrees of freedom $[64,65]$. Nevertheless, these refinements would require major extensions of the method and thus present a topic of future work.

\section{ACKNOWLEDGMENTS}

We would like to thank P. Van Isacker for providing us with the computer program IBM-1. K.N. acknowledges support from the Japan Society for the Promotion of Science. This work has been supported in part by the QuantiXLie Centre of Excellence. The work of L.M.R. was supported by Spanish MINECO Grants No. FPA2015-65929-P MINECO and No. FIS2015-63770-P MINECO.
[1] A. Bohr and B. M. Mottelsson, Nuclear Structure, Vol. 2 (Benjamin, New York, 1975), p. 45.

[2] R. F. Casten, Nuclear Structure from a Simple Perspective (Oxford University Press, Oxford, 2005).

[3] P. Cejnar, J. Jolie, and R. F. Casten, Rev. Mod. Phys. 82, 2155 (2010).

[4] G. Gürdal, E. A. Stefanova, P. Boutachkov, D. A. Torres, G. J. Kumbartzki, N. Benczer-Koller, Y. Y. Sharon, L. Zamick, S. J. Q. Robinson, T. Ahn, V. Anagnostatou, C. Bernards, M. Elvers, A. Heinz, G. Ilie, D. Radeck, D. Savran, V. Werner, and E. Williams, Phys. Rev. C 88, 014301 (2013).

[5] A. Corsi, J.-P. Delaroche, A. Obertelli, T. Baugher, D. Bazin, S. Boissinot, F. Flavigny, A. Gade, M. Girod, T. Glasmacher, G. F. Grinyer, W. Korten, J. Libert, J. Ljungvall, S. McDaniel, A. Ratkiewicz, A. Signoracci, R. Stroberg, B. Sulignano, and D. Weisshaar, Phys. Rev. C 88, 044311 (2013).

[6] Y. Toh, C. J. Chiara, E. A. McCutchan, W. B. Walters, R. V. F. Janssens, M. P. Carpenter, S. Zhu, R. Broda, B. Fornal, B. P. Kay, F. G. Kondev, W. Królas, T. Lauritsen, C. J. Lister, T. Pawłat, D. Seweryniak, I. Stefanescu, N. J. Stone, J. Wrzesiński, K. Higashiyama, and N. Yoshinaga, Phys. Rev. C 87, 041304 (2013).

[7] J. Sun, Z. Shi, X. Li, H. Hua, C. Xu, Q. Chen, S. Zhang, C. Song, J. Meng, X. Wu, S. Hu, H. Zhang, W. Liang, F. Xu, Z. Li, G. Li, C. He, Y. Zheng, Y. Ye, D. Jiang, Y. Cheng, C. He, R. Han, Z. Li, C. Li, H. Li, J. Wang, J. Liu, Y. Wu, P. Luo, S. Yao, B. Yu, X. Cao, and H. Sun, Phys. Lett. B 734, 308 (2014).

[8] N. Yoshinaga, K. Higashiyama, and P. H. Regan, Phys. Rev. C 78, 044320 (2008).

[9] M. Honma, T. Otsuka, T. Mizusaki, and M. Hjorth-Jensen, Phys. Rev. C 80, 064323 (2009).

[10] K. Kaneko, T. Mizusaki, Y. Sun, and S. Tazaki, Phys. Rev. C 92, 044331 (2015).

[11] L. Gaudefroy, A. Obertelli, S. Péru, N. Pillet, S. Hilaire, J. P. Delaroche, M. Girod, and J. Libert, Phys. Rev. C 80, 064313 (2009).

[12] T. Nikšić, P. Marević, and D. Vretenar, Phys. Rev. C 89, 044325 (2014).
[13] Z. H. Wang, J. Xiang, W. H. Long, and Z. P. Li, J. Phys. G: Nucl. Part. Phys. 42, 045108 (2015).

[14] P. Sarriguren, Phys. Rev. C 91, 044304 (2015).

[15] E. Padilla-Rodal, O. Castanos, R. Bijker, and A. GalindoUribarri, Rev. Mex. Fis. S 52, 57 (2006).

[16] J. Barea and F. Iachello, Phys. Rev. C 79, 044301 (2009).

[17] K. Heyde and J. L. Wood, Rev. Mod. Phys. 83, 1467 (2011).

[18] M. Bender, P.-H. Heenen, and P.-G. Reinhard, Rev. Mod. Phys. 75, 121 (2003).

[19] T. H. R. Skyrme, Nucl. Phys. 9, 615 (1958).

[20] J. Decharge, M. Girod, and D. Gogny, Phys. Lett. B 55, 361 (1975).

[21] D. Vretenar, A. V. Afanasjev, G. Lalazissis, and P. Ring, Phys. Rep. 409, 101 (2005).

[22] T. Nikšić, D. Vretenar, and P. Ring, Prog. Part. Nucl. Phys. 66, 519 (2011).

[23] H. Mei, J. Xiang, J. M. Yao, Z. P. Li, and J. Meng, Phys. Rev. C 85, 034321 (2012).

[24] T. R. Rodríguez, Phys. Rev. C 90, 034306 (2014).

[25] P. Ring and P. Schuck, The Nuclear Many-Body Problem (Springer-Verlag, Berlin, 1980).

[26] R. Rodríguez-Guzmán, J. L. Egido, and L. M. Robledo, Nucl. Phys. A 709, 201 (2002).

[27] Z. P. Li, T. Nikšić, and D. Vretenar, J. Phys. G: Nucl. Part. Phys. 43, 024005 (2016).

[28] A. Petrovici, K. Schmid, F. Grmmer, A. Faessler, and T. Horibata, Nucl. Phys. A 483, 317 (1988).

[29] A. Petrovici, K. Schmid, F. Grmmer, and A. Faessler, Nucl. Phys. A 504, 277 (1989).

[30] A. Petrovici, K. Schmid, F. Grmmer, and A. Faessler, Nucl. Phys. A 517, 108 (1990).

[31] A. Petrovici, E. Hammarn, K. Schmid, F. Grmmer, and A. Faessler, Nucl. Phys. A 549, 352 (1992).

[32] A. Petrovici, K. Schmid, and A. Faessler, Nucl. Phys. A 710, 246 (2002).

[33] K. Nomura, N. Shimizu, and T. Otsuka, Phys. Rev. Lett. 101, 142501 (2008).

[34] F. Iachello and A. Arima, The Interacting Boson Model (Cambridge University Press, Cambridge, 1987). 
[35] K. Nomura, T. Otsuka, and P. Van Isacker, J. Phys. G: Nucl. Part. Phys. 43, 024008 (2016).

[36] K. Nomura, R. Rodríguez-Guzmán, and L. M. Robledo, Phys. Rev. C 94, 044314 (2016).

[37] K. Nomura, D. Vretenar, T. Nikšić, and B.-N. Lu, Phys. Rev. C 89, 024312 (2014).

[38] K. Nomura, R. Rodríguez-Guzmán, and L. M. Robledo, Phys. Rev. C 92, 014312 (2015).

[39] K. Nomura, T. Nikšić, and D. Vretenar, Phys. Rev. C 93, 054305 (2016).

[40] P. Duval, D. Goutte, and M. Vergnes, Phys. Lett. B 124, 297 (1983).

[41] U. Kaup, C. Mönkemeyer, and P. v. Brentano, Z. Phys. A 310, 129 (1983).

[42] S. Goriely, S. Hilaire, M. Girod, and S. Péru, Phys. Rev. Lett. 102, 242501 (2009).

[43] J. F. Berger, M. Girod, and D. Gogny, Nucl. Phys. A 428, 23 (1984).

[44] L. M. Robledo, R. R. Rodríguez-Guzmán, and P. Sarriguren, Phys. Rev. C 78, 034314 (2008).

[45] R. Rodríguez-Guzmán, P. Sarriguren, L. M. Robledo, and J. E. García-Ramos, Phys. Rev. C 81, 024310 (2010).

[46] J. N. Ginocchio and M. W. Kirson, Nucl. Phys. A 350, 31 (1980).

[47] T. Otsuka, A. Arima, and F. Iachello, Nucl. Phys. A 309, 1 (1978).

[48] P. D. Duval and B. R. Barrett, Phys. Lett. B 100, 223 (1981).
[49] K. Nomura, N. Shimizu, D. Vretenar, T. Nikšić, and T. Otsuka, Phys. Rev. Lett. 108, 132501 (2012).

[50] A. Frank, P. Van Isacker, and C. E. Vargas, Phys. Rev. C 69, 034323 (2004).

[51] K. Nomura, R. Rodríguez-Guzmán, L. M. Robledo, and N. Shimizu, Phys. Rev. C 86, 034322 (2012).

[52] K. Nomura, R. Rodríguez-Guzmán, and L. M. Robledo, Phys. Rev. C 87, 064313 (2013).

[53] K. Nomura, N. Shimizu, and T. Otsuka, Phys. Rev. C 81, 044307 (2010).

[54] R. Bengtsson, T. Bengtsson, J. Dudek, G. Leander, W. Nazarewicz, and J.-Y. Zhang, Phys. Lett. B 183, 1 (1987).

[55] R. Bengtsson and W. Nazarewicz, Z. Phys. A 334, 269 (1989).

[56] W. Nazarewicz, Phys. Lett. B 305, 195 (1993).

[57] P. Van Isacker, computer program IBM-1 (unpublished).

[58] Brookhaven National Nuclear Data Center, http://www. nndc.bnl.gov

[59] T. Kibédi and R. Spear, At. Data Nucl. Data Tables 89, 77 (2005).

[60] J. Ljungvall, A. Görgen, M. Girod, J.-P. Delaroche, A. Dewald, C. Dossat, E. Farnea, W. Korten, B. Melon, R. Menegazzo, A. Obertelli, R. Orlandi, P. Petkov, T. Pissulla, S. Siem, R. P. Singh, J. Srebrny, C. Theisen, C. A. Ur, J. J. Valiente-Dobón, K. O. Zell, and M. Zielińska, Phys. Rev. Lett. 100, 102502 (2008).

[61] N. Stone, At. Data Nucl. Data Tables 90, 75 (2005).

[62] L. Wilets and M. Jean, Phys. Rev. 102, 788 (1956).

[63] A. S. Davydov and G. F. Filippov, Nucl. Phys. 8, 237 (1958).

[64] J. Elliot and A. White, Phys. Lett. B 97, 169 (1980).

[65] J. Elliot and J. Evans, Phys. Lett. B 101, 216 (1981). 